\title{
An Iterative Algorithm of Coupling the Kinetic Code for Plasma Periphery (KIPP) with SOLPS
}

\author{
Menglong Zhao ${ }^{\mathrm{a}, \mathrm{b}, *}$, Alex Chankin ${ }^{\mathrm{a}}$, David Coster $^{\mathrm{a}}$ \\ ${ }^{a}$ Max-Planck-Institut für Plasmaphysik, Garching bei München, Boltzmannstr. 2, 85748, \\ Germany \\ ${ }^{b}$ Physik-Department E28, Technische Universität München, 85747 Garching, Germany
}

\begin{abstract}
Power exhaust is one of the critical issues for future fusion devices, e.g. ITER. The calculation of power deposition is critical for the divertor design. SOLPS is the main tool for predictions of the Scrape-off Layer (SOL) and divertor conditions in the future fusion device ITER, where parallel kinetic effects in the SOL will play an important role. SOLPS uses a collisional fluid model which does not take kinetic effects into account. The present work has enabled SOLPS in its $1 \mathrm{D}$ version to incorporate electron kinetic effects by coupling it with the Kinetic Code for Plasma Periphery (KIPP). An iterative algorithm, which is made as an automatic process, is investigated in this work.
\end{abstract}

Keywords: SOLPS, KIPP, coupling, fluid model, kinetic effects, Scrape-off Layer

2010 MSC: 00-01, 99-00

\section{Introduction}

In future fusion devices e.g. ITER, approximately $80 \%$ of the heating power due to the fusion reaction should be exhausted in the divertor region, hence the divertor should be well designed to have the capability of handling such a large power exhaust.

\footnotetext{
*Corresponding author

Email address: menglong.zhao@ipp.mpg.de (Menglong Zhao)
}

Preprint submitted to Journal of ${ }^{A} T_{E} X$ Templates

September 28, 2018 
A prediction of the heat flux deposited on divertor targets is required for the divertor design. For present tokamak edge plasma simulation codes such as SOLPS [1], the 2D multi-fluid Braginskii model [2] is implemented. It is based on moments of the Vlasov-Fokker Planck equation $[3,4,5]$. The first three moments with unknown variables: ion and electron densities, velocities and temperatures, are commonly used in a fluid model with closure equations to close the hierarchical structure of moment equations. The closure equations are the relations of the higher order terms (the undetermined terms: ion and electron heat flux densities, thermal force coefficient) and the variables to be solved for. In the Braginskii model, the closure of moment equations is achieved by solving Vlasov-Fokker-Planck equation based on the assumption that the distribution function deviates only slightly from the Maxwellian due to the presence of gradients of density and temperature. However, with respect to the electron parallel transport in the SOL, this assumption is easily violated since the electron distribution function in particular near the target is, in most cases, far away from the Maxwellian, being highly asymmetric due to the presence of super-thermal electrons which are much less collisional and carry the bulk of the heat flux $[6,7,8,9]$. Hence they contribute to the extended tails of down-streaming electrons near the target. The sheath potential drop, acting as an energy filter, reflects low energy electrons, thus cutting off the upstreaming electron distribution function [10]. In addition, parallel electron transport is non-local due to long mean free paths of super-thermal electrons $[9,11,12]$.

Earlier studies attempted to simplify the kinetic equation in the superthermal limit $[13,12,14,15]$ by only considering the collisions of super-thermal electrons which are responsible for the heat transport and then to obtain a parallel heat transport equation with non-local terms which can be easily solved numerically. For other analytical studies, self-similar solutions [11, 16, 17] for the kinetic equation with a simplified collision operator are searched in order to obtain an analytical expression for parallel conductive heat flux density which converges to Braginskii formula in the collisional limit. These analytical formulas clarified the dependence of parallel conductive heat flux density on other 
macroscopic parameters despite many limitations posed on their applications by the prerequisite assumptions.

Another approach of including non-local effects is to close the hierarchical structure in a higher moment (the 4th moment) [18, 19, 20, 21, 22, 23, 24, 25, 26]. A higher order of a macroscopic parameter $\left\langle v^{4}\right\rangle$ is introduced, but it cannot be determined self-consistently. This parameter $\left\langle v^{4}\right\rangle$ is then closed by being related to lower order parameters by assuming a bi-Maxwellian distribution function [27].

Some other non-local expressions for the heat flux density are also proposed in various kinetic simulations $[28,29,30,31]$. They take into account the nonlocal nature of the conductive heat flux density by incorporating heat flux densities at all positions into one i.e. calculating the heat flux density at one certain location based on the whole parameter profiles, not just local values and their derivatives.

The studies mentioned above are all within the framework of a fluid model. However, from kinetic simulations $[32,33,34,35,36,37]$ it was found that the electron distribution function near the target deviates significantly from the Maxwellian due to super-thermal electrons coming from upstream. Therefore kinetic simulations are necessary to elucidate the physics that the fluid model is not able to cover. The full kinetic equation is often reduced to a gyro-kinetic one by averaging the gyro-motion.

Two main approaches are adopted to solve the kinetic equation: particle-incell (PIC) [38] and continuum [39] methods.

Particle in cell (PIC) method. In the PIC approach, the initial distribution function in the kinetic equation is recovered by a number of "macroparticles" statistically, each of which consists of many real particles. Then the evolution of the distribution function is represented by evolving the positions and velocities of the "macroparticles". This approach is numerically easier to implement in a code but it requires a large number of "macroparticles" to reduce the statistical noise. Some codes have been utilizing this method to investigate kinetic effects 
in edge plasmas, e.g. PARASOL [40, 41, 42, 43, 44, 45, 34, 46, 47, 48], BIT [49, $50,51,52,53,54,55,56,57], \mathrm{W} 1$ and $\mathrm{W} 2[58,59,60]$.

Continuum method. In the continuum approach utilized in other codes: FPET [35], ALLA [33], COGENT [61, 62, 63], the kinetic equation is solved in a discretized phase space. The macroscopic parameters are easily calculated by taking moments of the distribution function. However, the simulation accuracy is limited by the resolution of velocity space grids that should be discretized in such a way so as to cover the wide range of temperatures along field lines.

Implementation of the simplified BGK collision operator [64] which is numerically and physically simpler than the Fokker-Planck one [65], allows one to couple kinetic effects related to the long mean free path particles [66, 67, 68] and non-local boundary conditions with a fluid model [69]. Nevertheless, the simplified approximation of a full collision operator is not applicable to arbitrary profiles of density and temperature because of its low accuracy and the lack of conservativeness [67]. The existing kinetic codes are either imperfect in including all the physical processes that already exist in a fluid code or extremely time-consuming. Therefore the Kinetic Code for Plasma Periphery (KIPP) $[70,71,72,73]$ was developed to investigate kinetic effects of parallel plasma transport in a systematic way with an aim of coupling it with SOLPS. It is supposed to be fast at a cost of sacrificing a few features but without losing accuracy of collision terms. On the other hand, SOLPS is a highly sophisticated code with self-consistent recycling and physical and chemical sputtering as well as atomic physics [74] which are the most sophisticated and time-consuming parts in the numerical implementation of a kinetic code. In this work, the so-

phisticated model of SOLPS and kinetic effects of electron parallel transport offered by KIPP are combined in the KIPP-SOLPS coupling algorithm [75].

\section{SOLPS}

SOLPS [1], an abbreviation for Scrape-off Layer Plasma Simulator, is a code package developed for the tokamak plasma edge physics community. It is a 


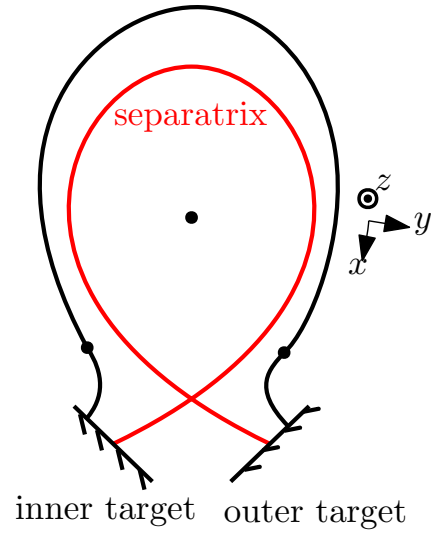

Figure 1: Poloidal cross section of a tokamak with lower single null. $x$ is along the poloidal direction from the inner target pointing to the outer target, $y$ is along the radial direction from the core pointing to the wall and $z$ is along the toroidal direction. All variables are assumed to be constant along $z$ in SOLPS

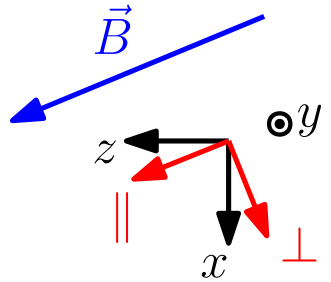

Figure 2: The magnetic field $\vec{B}$ in a tokamak has two components: toroidal field (TF) $B_{z}$ along the toroidal direction $z$ and poloidal field (PF) $B_{x}$ along the poloidal direction $x$. The parallel direction $\|$ is along the magnetic field while the perpendicular direction $\perp$ is perpendicular to both the parallel (\|) and radial (y) directions. Fluxes in the poloidal direction $(x)$ are actually sums of projections of parallel and perpendicular fluxes on the poloidal direction $(x)$.

2D fluid code solving Braginskii equations [2], which assumes that a tokamak device is toroidally symmetric, with poloidal direction from the inner target to the outer one denoted by $x$ and radial direction from the inner region to the vessel wall denoted by $y$, as shown in Fig. 1. The magnetic field has toroidal $\left(B_{z}\right)$ and poloidal $\left(B_{x}\right)$ components in a tokamak device. The transport in the poloidal direction $x$ in SOLPS arises due to the transport in the parallel $(\|)$ and perpendicular $(\perp)$ directions as shown in Fig. 2 (" $\perp$ " was called diamagnetic direction in [1]). Here and in later discussions the subscript $\perp$ denotes the perpendicular direction which is perpendicular to both the magnetic field and the radial direction $y$ (detailed discussions can be found in Appendix A).

We would like to remind again that, in later discussions, $x$ and $y$ indicate the poloidal and radial directions, respectively, while $\|$ and $\perp$ indicate the direction parallel to the magnetic field and the perpendicular direction which is perpendicular to both the parallel $(\|)$ and radial $(y)$ directions, respectively. And in later discussions, 'perpendicular' transport means the transport only in 
the perpendicular direction $(\perp)$.

SOLPS was created as a 2D code, with poloidal and radial directions based on the assumption that all parameters in the toroidal direction are constant. For a number of applications, a 1D version of SOLPS was created [76, 77]. Details of the 1D SOLPS adaptation can be found in the appendix. The kinetic code KIPP (described in detail in section 3.2) is mainly aimed at coupling parallel kinetic transport with SOLPS, therefore it is the 1D SOLPS version without currents and drifts, with variables only varying in the poloidal direction, that is used for testing the coupling algorithm (described in section 4).

Neutrals were originally treated kinetically in SOLPS due to their long mean free paths. However, for the following discussions and coupling tests, neutrals will be treated as a fluid since the 1D geometry is used in this work and the fluid treatment is computationally significantly faster. Additionally, the neutrals are not directly involved in the coupling algorithm.

\section{Electron kinetic effects and KIPP}

\subsection{Limitations of fluid models and kinetic factors}

This section describes four electron-related "kinetic factors" (this name was used in $[36,37])$ : electron heat conduction coefficient $c_{e}$, thermal force coefficient

$k_{\|}$, sheath potential drop $\Delta \phi$ and electron sheath heat transmission coefficient $\gamma_{e}$. Their specifications are necessary for numerical solutions of the fluid model since they cannot be determined self-consistently within the framework of the fluid model.

\subsubsection{Heat conduction and thermal force coefficients}

The closure equations for the Braginskii equations are:

$$
\begin{aligned}
q_{e \|}^{\text {cond }} & =-c_{e} n \tau_{e} \frac{T_{e}}{m_{e}} \nabla_{\|} T_{e} \\
R_{T_{\|}} & =-k_{\|} n \nabla_{\|} T_{e}
\end{aligned}
$$


where

$$
\begin{aligned}
& c_{e}=3.16 \\
& k_{\|}=0.71
\end{aligned}
$$

for singly charged ion. The closure equations are only valid under the assumption that the collisionality is high enough so that the electron conductive heat flux density and thermal force scale linearly with the local temperature gradient.

However, from previous 1D kinetic simulations [6, 7, 8, 9, 72], the conductive heat flux was found to be carried mostly by Heat Carrying Electrons (HCE) even in the collisional limit. According to [72], the maximum energy flux density is achieved at $v_{\|}=2.82 v_{\mathrm{th}}, v_{\perp}=1.98 v_{\mathrm{th}}$, corresponding to $5.95 T_{e}$ of the kinetic energy. The mean free path of HCE is $\lambda_{\mathrm{HCE}}=v_{\|} \times \tau_{\mathrm{HCE}} \approx 25 \lambda_{\mathrm{th}}$ [72]. HCE are experiencing much fewer collisions than thermal electrons. The collisional limit condition is often violated for HCE even when thermal electrons are collisional.

\subsubsection{Sheath potential drop and electron sheath heat transmission coefficient}

A thin layer called "Debye sheath" [78] is present in the region where the plasma interacts with the target. The sheath is so thin that it is usually collisionless and fully kinetic. Hence it cannot be described by the fluid model, as a result, the boundary conditions for the fluid equations (section 2) have to be specified separately. Here only the boundary kinetic factors for equations related to electrons are discussed: sheath potential drop $\Delta \phi$ for the charge conservation equation (Eq. (A.9)) and electron sheath heat transmission coefficient $\gamma_{e}$ for the electron energy conservation equation (Eq. (A.12)).

The boundary of the simulation domain at the target in SOLPS is technically the sheath edge instead of the target. The determination of $\Delta \phi$ and $\gamma_{e}$ depends on the local electron distribution function, which is unknown at the boundary. In the collisional limit, one often assumes that electrons have cut-off and half Maxwellian distributions at the sheath edge and the target, respectively 
to obtain the values of $\Delta \phi$ and $\gamma_{e}[10]$ :

$$
\begin{aligned}
\Delta \phi & \approx-3 \frac{T_{e t}}{e} \\
\gamma_{e} & =2+\frac{|e \Delta \phi|}{T_{e t}} \approx 5
\end{aligned}
$$

where $T_{e t}$ is target electron temperature.

Nevertheless, electrons at the sheath edge can hardly be Maxwellian or cutoff Maxwellian, especially in medium upstream collisionalities [10, 27, 79], since the electrons that overcome the sheath potential drop and contribute to the boundary heat flux may come from far upstream [79], being characterized by significantly higher temperature than the local one $[80,81,82,83,84,85,86,87$, $88,89]$ due to the temperature variation along a flux tube in the edge. Hence the prerequisite for deriving Eqs. (5) and (6) is not always valid in the edge. From previous kinetic simulations [36,37], it was found that the non-local effect can significantly influence values of $\gamma_{e}$ and $\Delta \phi$.

\subsection{Introduction to KIPP}

The Vlasov-Fokker-Planck equation is being solved in KIPP [70, 71]. At the present stage, the code is focusing on the electron parallel transport:

$$
\frac{\partial \tilde{f}_{e}}{\partial \tilde{t}}+\tilde{v_{\|}} \nabla_{\|} \tilde{f}_{e}-\tilde{E}_{\|} \frac{\partial \tilde{f}_{e}}{\partial \tilde{v}_{\|}}=\left(\frac{\partial \tilde{f}}{\partial \tilde{t}}\right)_{\text {coll. }}+\tilde{S_{\mathrm{E}}}+\tilde{S_{\mathrm{p}}}
$$

where the tilde sign " $\sim$ " denotes that all parameters appearing in this code are dimensionless, normalized by reference parameters: density $n_{0}$, temperature $T_{0}$, velocity $v_{0}$ and collision logarithm $\Lambda_{0}$, with $T_{0}=m_{e} v_{0}^{2}$, normally taken at the stagnation point. This sign will be omitted in the following discussions in this section. $f_{e}$ is a $3 \mathrm{D}$ distribution function with two dimensions in velocity space: parallel and gyro-averaged perpendicular velocity, and one dimension in physical space along the magnetic field. $S_{\mathrm{E}}$ and $S_{\mathrm{p}}$ are electron energy and particle sources respectively.

Eq. (7) is solved by using an operator splitting scheme [70], with parallel free-streaming for $1 / 2$ time step followed by Coulomb collisions and the electric 
field force over one time step, followed again by the other $1 / 2$ time step of freestreaming $[70,90,91]$. The numerical implementation of the terms in Eq. (7) can be found in [70].

\subsection{Boundary conditions}

Boundary for velocity space. KIPP is currently developed as a 3D (1D2V) code. As described above, all parameters are normalized by the reference parameters: $n_{0}, T_{0}, \Lambda_{0}, v_{0}$. The velocity grids are created based on the reference parameter $v_{0}$. The highest velocity resolved in all simulation cases is $v_{\max }=7 v_{0}$. Distribution functions outside of this range are treated as zero. The justification for this choice was given in [70].

Boundary for spatial space: the stagnation point. KIPP is currently designed for 1D space along the magnetic field line with the stagnation point and the target at the left and right ends, respectively. There are smax +1 cells created for the 1D space and they are numbered: $0,1, \cdots, \operatorname{smax}$. The left boundary is the center of cell 0 , assumed as the stagnation point where the reconstruction is based on the $1^{\text {st }}$ order upwind scheme. The numerical flux entering the boundary is assumed as:

$$
F_{-\frac{1}{2}}^{n}\left(v_{\|}, v_{\perp}\right)=-F_{\frac{1}{2}}^{n}\left(-v_{\|}, v_{\perp}\right)
$$

making the stagnation point a reflective boundary.

Boundary for spatial space: the target. The right boundary is the right face of cell smax, assumed to be the target (technically the sheath edge). For numerical simplicity, Poisson equation and hence the Debye sheath is not dealt with in KIPP since solving the Debye sheath requires a very small time step $\Delta t \sim \tau_{g}$ (the gyro-motion time) and a small spatial cell size $d s \sim L_{D}$ (the width of the Debye sheath). Instead, the logical sheath boundary condition is implemented [92], of which the main idea is to give a reasonable electron distribution function at the sheath edge without having to solve the sheath region of inherently small space and time scales. The results compare reasonably well with those of analytical 
analysis and those from simulations with solving the sheath region, since it captures the main physics of the sheath [92]. The logical sheath boundary condition is utilized to specify only the numerical flux entering the boundary $\left(v_{\|}<0\right)$ since the downstreaming flux $\left(v_{\|}>0\right)$ out of the final boundary is determined self-consistently by the upstream conditions. It is equivalent to the assumption that the distribution function at the simulation boundary is prescribed as:

$$
f_{t}\left(v_{\|}, v_{\perp}\right)= \begin{cases}f_{t}^{\text {down }}\left(v_{\|}, v_{\perp}\right) & \text { if } v_{\|}>0 \\ f_{t}^{\text {down }}\left(-v_{\|}, v_{\perp}\right) & \text { if }-v_{c} \leq v_{\|} \leq 0 \\ 0 & \text { if } v_{\|}<-v_{c}\end{cases}
$$

where $f_{t}$ is the distribution function at the boundary and $f_{t}^{\text {down }}$ is the downstreaming part.

\section{Iterative coupling of KIPP with SOLPS}

As discussed in section 3.1, the fluid description of electrons is not always valid in the SOL, kinetic corrections are necessary for arbitrary collisionalities. Since the fluid model in SOLPS is already highly sophisticated with selfconsistent recycling, physical and chemical sputtering, as well as atomic physics models, which are the most time-consuming parts of a kinetic code, KIPP was developed to account for kinetic effects of electron parallel transport in SOLPS, leaving the rest of the physical models intact.

One difficulty with coupling a kinetic code to a fluid one is that they have very different time scales. For a plasma with constant $T=50 \mathrm{eV}, n=1.0 \times$ $10^{19} \mathrm{~m}^{-3}$ along a $25 \mathrm{~m}$ flux tube, the characteristic time scale of the fluid model used in a fluid code is the upstream ion transport time $\sim 10^{-4} \mathrm{~s}$, however, the time scale of a kinetic code without resolving the gyro-motion can be characterized as the electron collision time at the target $\sim 10^{-7} \mathrm{~s}$. The direct real-time coupling is obviously not possible [93]. One can think of an iterative coupling algorithm instead, which may offer a possible way of coupling a kinetic code to 
a fluid one. In the following sections, an iterative coupling algorithm will be investigated.

One iterative coupling scheme, which avoids the difficulty caused by different time scales, is proposed here. SOLPS passes converged profiles of macroscopic plasma parameters to KIPP, while KIPP passes effective kinetic factors back to SOLPS. This process is repeated until coupling onvergence is reached. The flowchart of this process is shown in Fig. 3. It consists of 4 main steps:

1. At the start of a coupled case, run SOLPS with default electron heat conduction coefficients $\left(c_{e}=3.16\right)$, thermal force coefficients $\left(k_{\|}=0.71\right)$, sheath potential drop (Eq. 5) and electron sheath heat transmission coefficient (Eq. 6). For a continuation of a coupled case, run SOLPS with the modified (effective) electron heat conduction coefficients $\left(c_{e f f}\right)$, thermal force coefficients $\left(k_{e f f}\right)$, sheath potential drop $\left(\Delta \phi_{e f f}\right)$ and electron sheath heat transmission coefficient $\left(\gamma_{e f f}\right)$. Calculations of the effective kinetic factors will be given below.

2. Transfer profiles of electron density, velocity and temperature, ion density and temperature, particle flux density from SOLPS to KIPP.

3. Maintaining the transferred profiles by automatic energy and particle sources, run KIPP and obtain new effective electron heat conduction coefficients $\left(c_{e f f}\right)$, thermal force coefficients $\left(k_{e f f}\right)$, sheath potential drop $\left(\Delta \phi_{e f f}\right)$ and electron sheath heat transmission coefficient $\left(\gamma_{e f f}\right)$.

4. Check if the coupling convergence has been reached: if yes, output all profiles and coefficients; if not, transfer kinetic factors back to SOLPS and continue with Step 1.

The iterative coupling is automatically carried out by some user-specified parameters. Analytical formulas of the modifications of the kinetic factors by KIPP are described in Appendix B. The above mentioned Step 3 is the most challenging since fluid equations in SOLPS and the kinetic equation in KIPP are not explicitly related to each other. The correspondence between terms involved to calculate the effective kinetic factors is clarified in Appendix B.1. 


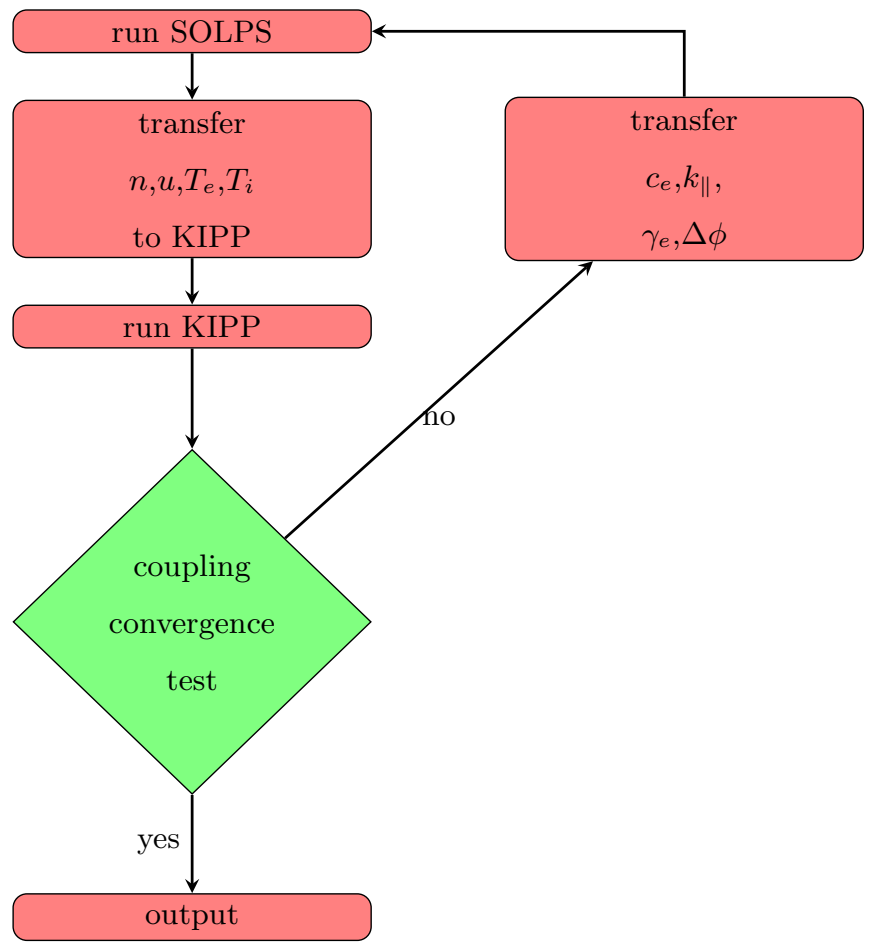

Figure 3: The schematic of the KIPP-SOLPS coupling algorithm.

More numerical details of the coupling algorithm can be found in Appendix B where it first compares equations solved in SOLPS and KIPP separately and then derives formulas for calculating effective kinetic factors in KIPP.

\section{Coupling simulation}

\subsection{The simulation geometry}

Since we are currently mainly focusing on kinetic effects of electron parallel transport, 1D SOLPS (Appendix A) is used here in order to test the compatibility of the coupling algorithm with the KIPP code.

In ASDEX-Upgrade, major plasma radius $R_{0}=1.65 \mathrm{~m}$, minor horizontal plasma radius $a=0.5 \sim 0.52 \mathrm{~m}$, minor vertical plasma radius is $\sim 0.8 \mathrm{~m}$ and the ellipticity is $\sim 1.8$. Therefore the perimeter of the plasma in the poloidal plane 
is $\sim 4.5 \mathrm{~m}$. We assume that the average distance along the poloidal magnetic field between the two targets is $\sim 5 \mathrm{~m}$. Half of the length is taken as the length of the simulation domain in our case since one target is assumed at one end, while the other is the stagnation point, $L_{p o l}=2.5 \mathrm{~m}$. The magnetic field $B=$ const, poloidal $B_{\text {pol }}=1.045285 \times 10^{-1} \mathrm{~T}$, toroidal $B_{\text {tor }}=9.945219 \times 10^{-1} \mathrm{~T}$.

SOLPS and KIPP generate simulation grids separately, therefore, the grids in both codes should be generated based on the above mentioned parameters and made consistent with each other.

As mentioned in section 3.2, all parameters in KIPP are dimensionless. However, in later discussions, all parameters in KIPP and SOLPS will be given in dimensional form unless otherwise stated.

\subsubsection{Grid cells generated in KIPP}

In the KIPP code, the 1D grid cells are generated along the magnetic field based on parameters EPSS and $L_{\text {par }}$ :

$$
\begin{aligned}
E P S S & =\frac{d s(m)}{d s(m+1)} \quad(0 \leq m<\operatorname{smax}) \\
L_{p a r} & =\sum_{m=0}^{s m a x} d s(m)
\end{aligned}
$$

where $m$ is the cell number and smax is the highest cell number, hence there are $\operatorname{smax}+1$ cells. $d s(m)$ is the parallel length of cell $m$ and $L_{p a r}$ is the length of the simulation domain along the magnetic field line:

$$
L_{p a r}=L_{p o l} \times \frac{\sqrt{B_{p o l}^{2}+B_{t o r}^{2}}}{B_{p o l}}
$$

The simulation domain in KIPP is shown as the red line in Fig. 4. The left end is the center of cell 0 , the stagnation point, therefore only half of cell 0 belongs to the simulation domain, the other half is assumed to have mirrorreflected parameters. The height of the cells in Fig. 4 doesn't reflect the real grids since the real geometry in KIPP is only $1 \mathrm{D}$ with boundaries at the two ends. Electron temperature, density, velocity, electric field, volumetric power and particle sources or sinks are defined at cell centers, while all fluxes are defined at cell faces. 


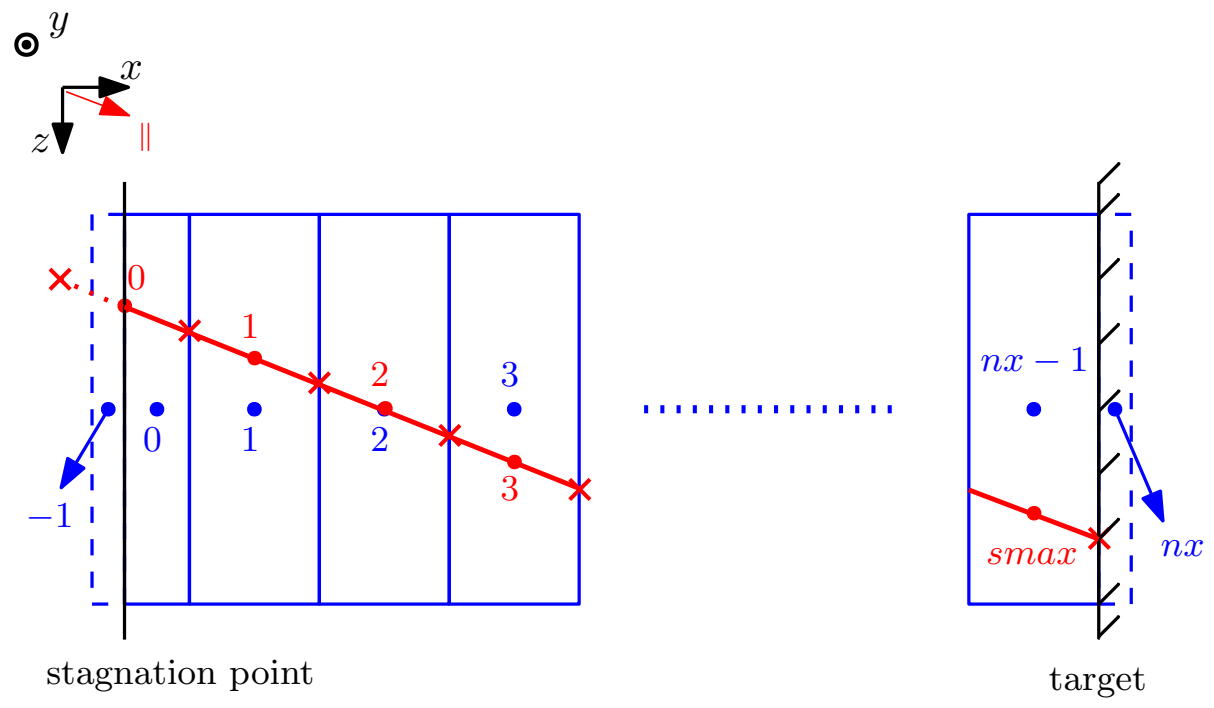

Figure 4: The grids generated in KIPP (red lines) and SOLPS (blue boxes). $x$ and $y$ are poloidal and radial directions respectively. The KIPP grid cells are generated along the direction parallel to the magnetic field with the center of cell 0 being the stagnation point and the right face of cell smax being the target, while the SOLPS grid cells are generated along the poloidal direction with two guard cells attached to the two ends. $n x=\operatorname{smax}+1$. The KIPP simulation domain extends from the stagnation point (the black solid line at the left end) to the target (the black solid line at the right end).

\subsubsection{Grid cells generated in SOLPS}

In contrast to KIPP, in SOLPS grid cells are generated along the poloidal direction. In order to be compatible with the KIPP grid, they are generated in the following way:

$$
\begin{aligned}
h x(0) & =\frac{1}{2} d s(0) \cdot b_{x} \\
h x(m) & =d s(m) \cdot b_{x} \quad(0 \leq m \leq n x-1)
\end{aligned}
$$

where $h x(m)$ is the poloidal length of the cell $m$ and $b_{x}=\frac{B_{p o l}}{\sqrt{B_{p o l}^{2}+B_{t o l}^{2}}} \cdot n x$ is the number of cells generated in the simulation domain.

$$
n x=\operatorname{smax}+1
$$


There are also two small guard cells attached to the two ends, numbered -1 and $n x$.

$$
\begin{aligned}
h x(-1) & =\frac{1}{1000} h x(0) \\
h x(n x) & =\frac{1}{1000} h x(n x-1)
\end{aligned}
$$

The guard cells are created only for implementing boundary conditions, and one should note that they are not included in the simulation domain. The comparison between the generated SOLPS and KIPP grids is shown in Fig. 4. The west and east boundaries (see Fig. A.26) are the left and right ends of the KIPP grid, respectively.

\subsection{Coupling setup}

The simulation geometry is defined in section 5.1, which physically corresponds to a flux tube from the upstream mid-plane position to the target with constant magnetic field projected onto the poloidal plane. The external power source is distributed evenly over cells 0 to 10 (from $x=0 \mathrm{~m}$ to $x \approx 0.83 \mathrm{~m}$ ). In order to systematically study the feasibility and performance of the iterative coupling algorithm, a series of cases with only deuterium plasma is run.

A pure deuterium plasma is used for the convergence and density scan studies in sections 5.3 and 5.4 respectively. Carbon is introduced as an impurity to study the case with large temperature drop but comparatively low upstream collisionality in section 6.2. $\alpha$ denotes one kind of species. It can be $D^{0}$ or $D^{+}$ for the pure deuterium plasma, while it can be $D^{0}, D^{+}, C^{0}, C^{+}, C^{2+}, C^{3+}$, $C^{4+}, C^{5+}$ or $C^{6+}$ for the deuterium plasma with the carbon impurity. The recycling coefficient at the target is set as 0.999 for deuterium, 0 for carbon. At the stagnation point, a constant deuterium ion density is maintained by an automatic particle source, while the deuterium and carbon neutral particle fluxes are specified as 0 , meaning that this is a reflective boundary for neutrals. The quasi-neutrality condition is fulfilled at each time step, indicating that electron density is evolving along with ion densities to satisfy Eq. (A.8). At the target, 
the velocity of each ion species satisfies the Bohm condition, but the speed of neutrals is assumed to be zero.

Ambipolar flows are specified through the whole simulation region. Since only electrons are treated kinetically, the default boundary condition for the ion energy conservation equation is used by specifying the ion sheath heat transmission coefficient [94]:

$$
\gamma_{i}=2.5
$$

This value is fixed during the entire run. As pointed out above, the adapted boundary condition for electron energy conservation equation is used. In the initial iteration, we specify [94]:

$$
\gamma_{e \|}^{\mathrm{ini}}=1.9+\left|e \Delta \phi^{\mathrm{ini}}\right| / T_{e t}
$$

Afterwards, this value is replaced by $\gamma_{e f f}$ calculated from Eq. (B.33). The initial sheath potential drop $\Delta \phi^{\text {ini }}$ is determined by Eq. (5) and then replaced by Eq. (B.21). Likewise, the coefficients for electron heat conduction and thermal force are initially specified as:

$$
\begin{aligned}
& c_{e}^{\mathrm{ini}}=3.16 \\
& k_{\|}^{\mathrm{ini}}=0.71
\end{aligned}
$$

and later replaced by $c_{e f f}$ and $k_{\text {eff }}$ calculated by Eqs. (B.19) and (B.16).

Initial plasma parameter profiles in KIPP are transferred from the steady state of the initial SOLPS run. Electron distribution functions in each cell are specified initially as Maxwellian. They evolve during KIPP runs to reach a quasi-steady state. However, the concept of the quasi-steady state is not well defined, so it cannot be regarded as a convergence criteria. In practice, one can run KIPP for a certain number of steps to reach a state at which the profile of an evolving parameter $P$ is changing slowly with time, for example,

$$
\left|\frac{d P^{n}}{d t}\right| \leq \beta\left|\frac{d P^{1}}{d t}\right|
$$


where $d t$ is the time step, $d P^{n}$ is the change of the parameter $P$ during the time step $\mathrm{n}$, and $\beta$ is a small free parameter. In order to get precise results in KIPP, the specified time step is chosen to be $[70,71]$ :

$$
d t \leq 0.1 \tau_{0}
$$

where $\tau_{0}$ is the Trubnikov collision time [65] in any cell. Because the collision time is typically smallest at the target, the condition (Eq. (23)) becomes

$$
d t \leq 0.1 \tau_{t}
$$

The evolving parameter $P$ here can be electron heat flux through cell faces or kinetic factors, but it cannot be electron density or temperature since these profiles are maintained in KIPP, not evolving with time. If $\beta=0$, it means that a complete steady state is reached, however, this is not possible for a kinetic code. Nevertheless, based on the simulation results shown in section 5.4, the exact value of $\beta$ seems not to influence the coupling steady state profiles, it rather influences the efficiency of reaching the coupling steady state. The study of the sensitivity of the efficiency of achieving a coupling steady state to $\beta$ is beyond the current work.

\subsection{Convergence study}

\subsubsection{Sensitivity to coupling schemes}

In SOLPS the electron heat conduction coefficient $c_{e}$ is defined at cell centers while the conductive heat flux density is defined at cell faces and then calculated with a hybrid regime based on interpolations of $c_{e}$ on cell faces. Since the numerical scheme implemented in SOLPS has been extensively studied and optimized, one should keep it intact. In KIPP the distribution function $f_{e}$ is defined at cell centers while the flux term is defined at cell faces (see section 3.2). One must therefore make changes to the numerical schemes of either SOLPS or KIPP. Three coupling schemes with increasing changes in SOLPS are investigated:

Scheme A 'center': replace $c_{e}$ in cell centers in the SOLPS part with the effective $c_{e f f}$ calculated in cell centers in the KIPP part. 
Scheme B 'face': replace the interpolated $c_{e}$ on cell faces in the SOLPS part with the effective $c_{e f f}$ calculated at cell faces in the KIPP part.

Scheme C 'decouple': replace the electron heat flux formula in the SOLPS part with decoupled convective and conductive pieces, making sure that the electron heat flux at each cell face is the same as the one calculated in KIPP in the coupling steady state.

The pure deuterium plasma with the stagnation point density $n_{u}=1.5 \times$ $10^{19} \mathrm{~m}^{-3}$ is used to test the coupling schemes. The steady state profiles from the three coupling schemes are compared in Fig. 5. As expected, they coincide with each other. In the following test simulations, the Scheme $\mathrm{C}$ is used to force the parallel electron heat flux densities in SOLPS and KIPP to have the same form in order to make the testing as simple as possible. However, in future applications, the Scheme B is proposed because it attempts to keep the numerical form of the heat flux density in SOLPS intact.

\subsubsection{Sensitivity to initial conditions}

In a coupling run, the KIPP part depends on profiles provided by the SOLPS part. If the iterative coupling scheme works, different initial conditions with different specifications in the SOLPS part are supposed to give the same profiles in the coupling steady state. There are two main free parameters in SOLPS determining electron profiles: electron heat flux limiter $\boldsymbol{\alpha}_{\boldsymbol{e}}$ and electron sheath heat transmission coefficient $\boldsymbol{\gamma}_{\boldsymbol{e} \|}$. The first coefficient $\left(\gamma_{e \|}\right)$ modifies the electron

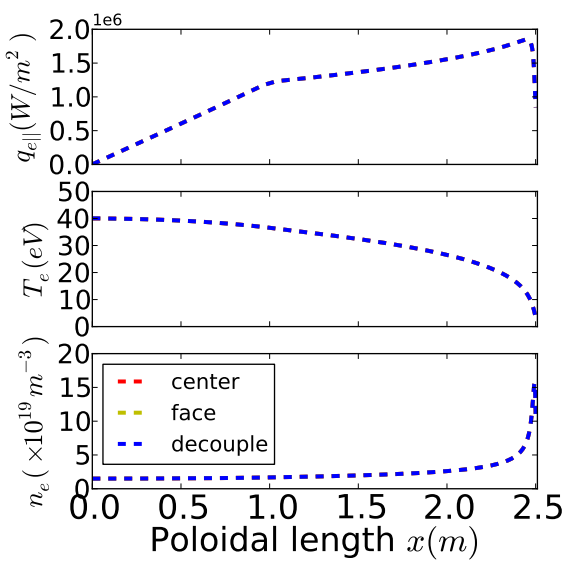

Figure 5: Three coupling schemes are compared. 'center', 'face', 'decouple' denote the coupling Schemes A, B, and C respectively. parallel heat flux density according 
to:

$$
q_{e \|}=\frac{5}{2} \Gamma_{e \|} T_{e}-\frac{1}{1+\frac{c_{e} \lambda_{e}}{\alpha_{e} L_{T_{e}}}} c_{e} \frac{n_{e} T_{e} \tau_{e}}{m_{e}} \nabla_{\|} T_{e}
$$

where $\lambda_{e}$ is electron mean free path and $L_{T_{e}}$ is electron temperature scale length. The latter determines the boundary electron heat flux density as discussed above. Four cases with different combinations of initial values of $\alpha_{e}$ and $\gamma_{e \|}$ are run to investigate coupling properties:

Case A Initial run with $\gamma_{e \|}=1.9+\left|e \Delta \phi^{\mathrm{ini}}\right| / T_{e t}, \alpha_{e}=\infty$.

Case B Initial run with $\gamma_{e \|}=4.1+\left|e \Delta \phi^{\text {ini }}\right| / T_{e t}, \alpha_{e}=\infty$.

Case C Initial run with $\gamma_{e \|}=1.9+\left|e \Delta \phi^{\mathrm{ini}}\right| / T_{e t}, \alpha_{e}=0.3$.

Case D Initial run with $\gamma_{e \|}=4.1+\left|e \Delta \phi^{\mathrm{ini}}\right| / T_{e t}, \alpha_{e}=0.3$.

The heat flux limiter is removed after the initial run of SOLPS. The coupling

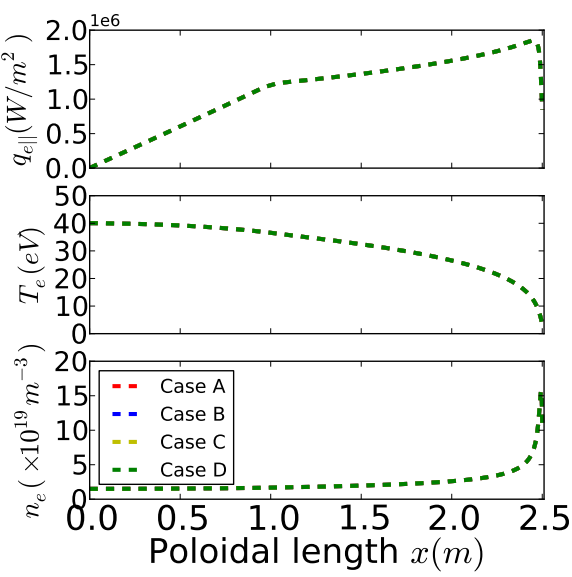

Figure 6: Four cases: A, B, C, D (see text for details) with different initial specifications finally converge to the same steady state profiles. However the coupling efficiency varies (see Fig. 7).

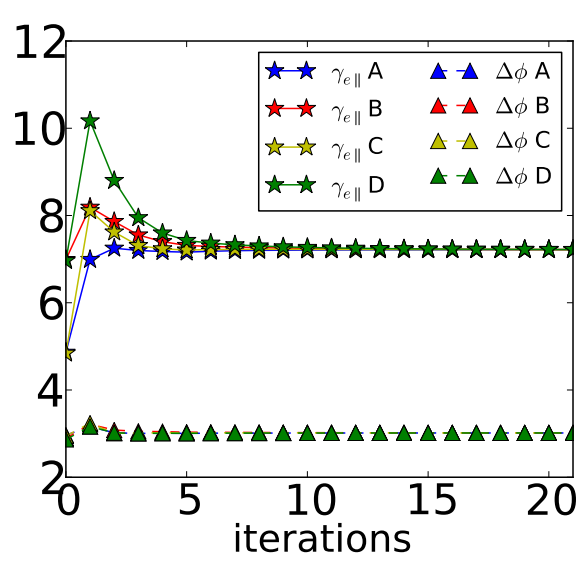

Figure 7: Evolution of $\gamma_{e \|}$ and $\Delta \phi$ with the coupling iterations for the four cases. Iteration $=0$ means the values used in the initial run of SOLPS; iteration=1 means the values obtained after the initial run of SOLPS and then KIPP, and the same for iteration $=$ $2,3, \cdots$. They converge to the same values (7.2 and 3.0 respectively). 
iterations converge to the same steady state profiles as expected, as shown in Fig. 6. This means that results of the iterative coupling scheme doesn't depend on initial conditions. However, the convergence efficiency is better when initial profiles are closer to the steady state profiles (see Fig. 7). In the following simulations, the initial specifications of Case A are used.

\subsection{Deuterium density scan study}

As pointed out in references $[32,27]$, the upstream collisionality $\nu *$ is the critical parameter that determines the role of kinetic effects of parallel electron propagation. Since $\nu * \propto T_{u}^{2} / n_{u}$, the easiest way of scanning upstream collisionality is to vary upstream plasma density while keeping the same power input (keeping the upstream temperature approximately constant). In this section, a pure deuterium plasma is assumed. A series of runs scanning the stagnation point ion density from $n_{u}=0.5 \times 10^{19} \mathrm{~m}^{-3}$ to $n_{u}=2.5 \times 10^{19} \mathrm{~m}^{-3}$ is carried out. The recycling coefficient and boundary conditions are the same as those described in section 5.2.

\subsubsection{Low stagnation point density $n_{u}=0.5 \times 10^{19} \mathrm{~m}^{-3}$}

The case with the stagnation point density $n_{u}=0.5 \times 10^{19} \mathrm{~m}^{-3}$ is run. The upstream collisionality $\left(\nu^{*}\right)$ of this case is $\approx 5.6$, which is very low. The evolution of electron density and temperature profiles with coupling iterations is shown in Fig. 8. One complete coupling iteration means that SOLPS runs until the steady state is achieved and then KIPP runs to the quasi-steady state based on density and temperature profiles transferred from the steady state SOLPS solutions. The evolution details are not clearly seen in this figure. Four locations: 1, 2, 3, 4 at poloidal coordinates: $x \approx 0.890 \mathrm{~m}, x \approx 1.514 \mathrm{~m}, x \approx 2.485 \mathrm{~m}, x \approx 2.499 \mathrm{~m}$, denoted by the four black vertical dashed lines are chosen to show evolution trends of some parameters.

$T_{e}$ at the four locations, the electron sheath heat transmission coefficient $\left(\gamma_{e \|}\right)$ and the sheath potential drop $(\Delta \phi)$ achieve the coupling steady state at iteration $=4$, as shown in Figs. 9 and 10a. $\Delta \phi$ is insensitive to electron kinetic 


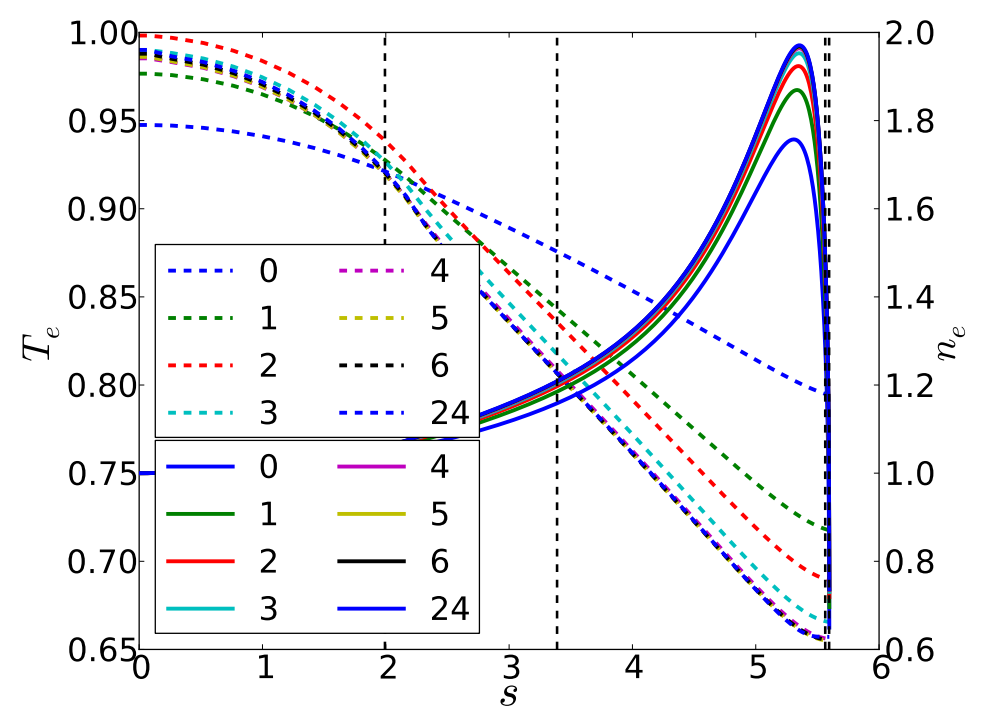

Figure 8: Evolution of electron density and temperature profiles for the case with the stagnation point density $n_{u}=0.5 \times 10^{19} \mathrm{~m}^{-3}$. All parameters are normalized by the reference parameters (density $n_{0}=0.5 \times 10^{19} \mathrm{~m}^{-3}$, the temperature $T_{0}=47 \mathrm{eV}$, Coulomb logarithm $\Lambda_{0}=15$ ). The horizontal axis $s$ is the parallel distance from the stagnation point (normalized by the electron mean free path $\lambda_{0}$ calculated based on the reference parameters). The colorful dashed (solid) lines are the profiles of electron temperature (density) evolving with coupling iterations. Profiles denoted by ' 0 ' means the convergent profiles after the initial SOLPS run based on default inputs. ' 1 ' means the convergent profiles achieved in SOLPS with the effective $c_{e f f}, \gamma_{e f f}, k_{e f f}, \Delta \phi_{e f f}$ which are calculated in KIPP based on the profiles with ' 0 '. The four vertical black dashed lines from left to right denote locations 1, 2, 3, 4 respectively.

effects at this collisionality. $\gamma_{e \|}$ in the coupling steady state is not far away from value 5 . This is due to the fact that the electron temperature profile is rather flat $\left(T_{0} / T_{t}<2\right)$. The electron distribution function has a slightly extended high energy tail, as shown in Fig. 11, where the 1D distribution function is defined by integrating $f_{e}\left(v_{\|}, v_{\perp}\right)$ over perpendicular velocity space as:

$$
F\left(v_{\|}\right)=\int f_{e}\left(v_{\|}, v_{\perp}\right) d \overrightarrow{v_{\perp}}
$$

The small deviation from the local Maxwellian at the high positive parallel velocity is responsible for the small increase (above 5) of the electron sheath 


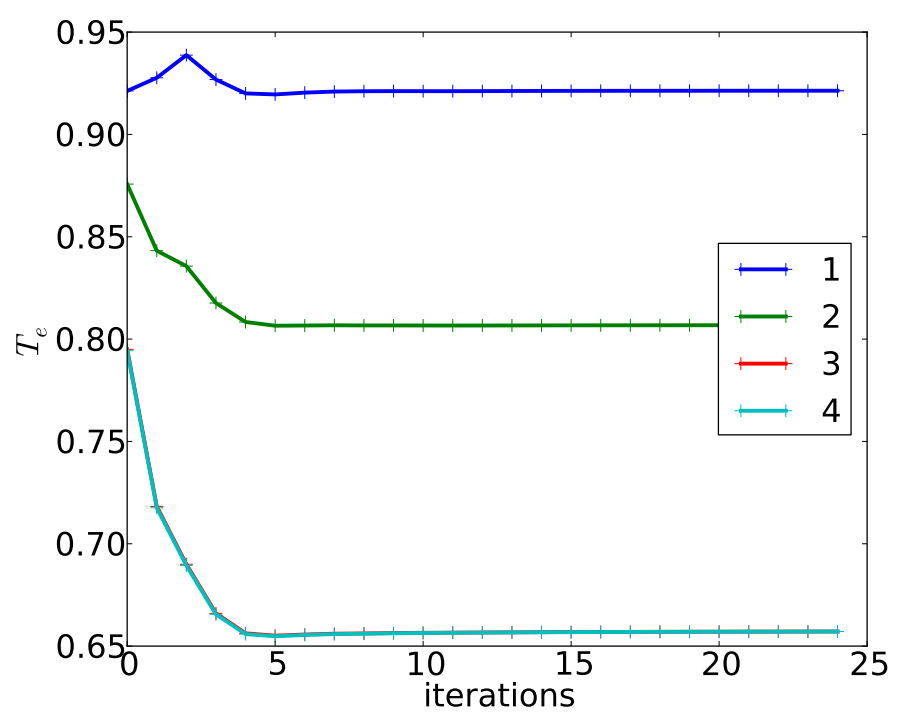

Figure 9: Evolution of the electron temperature with coupling iterations at the 4 locations: 1, 2, 3, 4 marked in Fig. 8.

heat transmission coefficient.
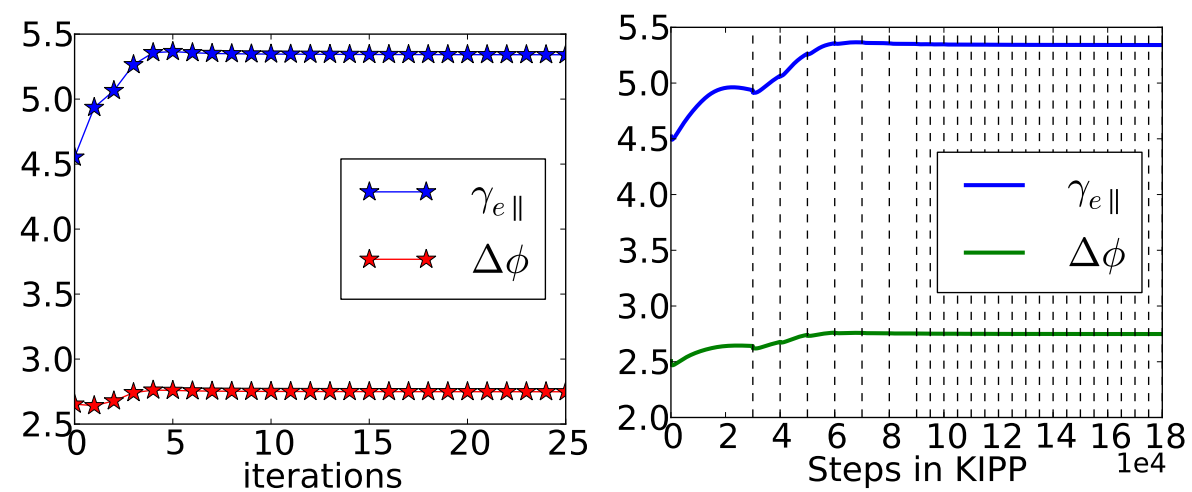

(a) Evolution of $\gamma_{e \|}$ and $\Delta \phi$ with coupling (b) Evolution of $\gamma_{e \|}$ and $\Delta \phi$ within KIPP. iterations.

Figure 10: Evolution of $\gamma_{e \|}$ and $\Delta \phi$ for the case with the stagnation point density $n_{u}=$ $0.5 \times 10^{19} \mathrm{~m}^{-3}$. 


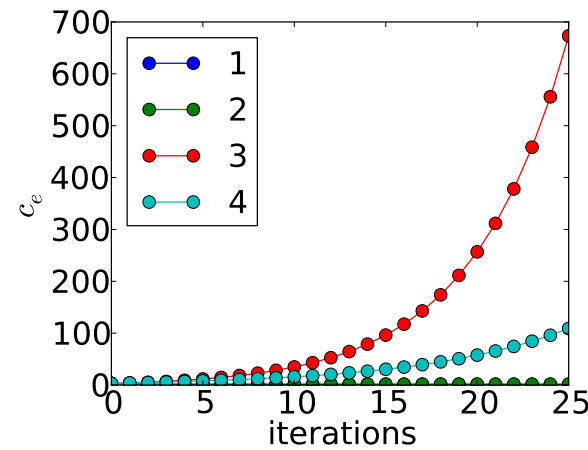

(a) Evolution of $c_{e}$ with coupling iterations at the 4 locations.

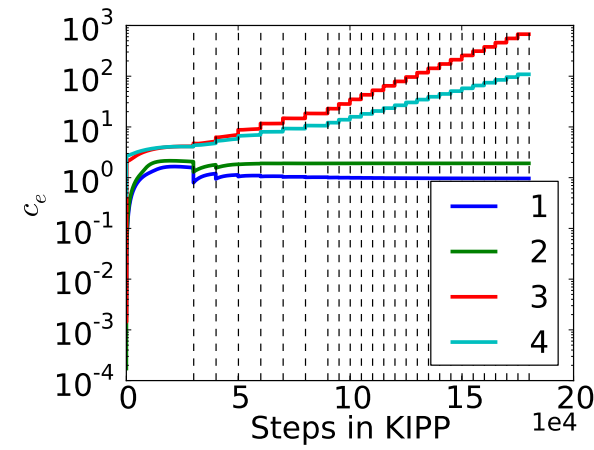

(b) Evolution of $c_{e}$ within KIPP at the 4 locations.

Figure 12: Evolution of $c_{e}$ within KIPP at the 4 locations for the case with the stagnation point density $n_{u}=0.5 \times 10^{19} \mathrm{~m}^{-3}$.

As mentioned in section 5.2, the quasi steady state is expected for each KIPP run. However, $\gamma_{e \|}$ doesn't fully reach the steady state in the second and third KIPP runs shown in Fig. 10b. The vertical dashed lines denote steps at which SOLPS is run and thus the maintained density and temperature profiles in KIPP are updated. The values at the first dashed line are corresponding to the values at iteration $=1$ in Fig. 12a, etc. It is not practical to set the quasisteady state as the exit condition for KIPP since one has no prior knowledge about the evolution trend and the time required to reach the quasi-steady state. At least one should assign more time steps at the initial iteration where the most significant changes are expected. The influence of the size and number of the time steps specified in each KIPP run on the optimization of the coupling regime might require further study. It is inferred however that the coupling steady state does not depend on these details.

Although profiles of $T_{e}$ and $n_{e}, \gamma_{e \|}$ and $\Delta \phi$ achieve the coupling steady state at iteration $=4$, as discussed above, $c_{e}$ at locations 3 and 4 (close to the target in Fig. 8) are increasing exponentially (numerical instability) with coupling iterations (see Fig. 12). 


\subsubsection{The numerical instability in the low upstream density case}

An exponential increase in $c_{e}$ reveals the limitation of the coupling scheme. Here we investigate the reason for this phenomenon, and a new coupling scheme is discussed later in section 5.5.

The vertical dashed lines in Fig. $12 \mathrm{~b}$ have the same meaning as those in Fig. 10b. The exponential increases of $c_{e}$ are seen at locations 3 and 4 (see Fig. 12a) after iteration $=$ 4 , although profiles of $T_{e}$ and $n_{e}, \gamma_{e} \|$

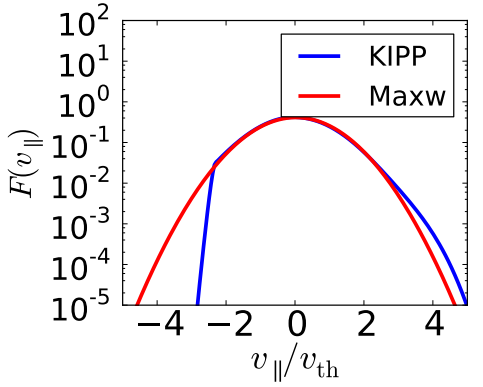

Figure 11: 1D electron distribution function at the cell adjacent to the target. The blue curve is the distribution function from KIPP while the red one is the local Maxwellian distribution function. and $\Delta \phi$ already achieve the coupling steady state. Changes in $c_{e}$ only occur at the initial step, afterwards they stay unchanged for the following steps in each KIPP run. This results in a flatter electron temperature profile, which, in turn, leads to larger coefficients for the next KIPP run. With coupling iterations, $c_{e}$ in this region keep increasing while electron temperature profile becomes increasingly flatter, creating a positive feedback loop, however, $c_{e} \nabla_{\|} T_{e} \approx$ const. Since the electron temperature profile is very flat in the region near the target, a flatter profile has no impact on any other parameters. This explains why the coupling steady state profiles of $T_{e}, n_{e}$ and $\gamma_{e \|}, \Delta \phi$ can be achieved, while the numerical instability still exists.

The instability might be explained by the fact that the prerequisite for a fluid model is violated in the region near the target for such a low collisionality case since the distribution function in this region deviates far from a Maxwellian one due to non-local transport [32]. The Braginskii formula for the electron conductive heat flux density completely fails. A new coupling scheme is proposed and discussed in section 5.5 in order to avoid such numerical instability in the low collisionality case. 
5.4.3. Medium stagnation point density $n_{u}=1.0-2.0 \times 10^{19} \mathrm{~m}^{-3}$

Here we analyse the case with the stagnation point density $n_{u}=1.5 \times$ $10^{19} \mathrm{~m}^{-3}$ which is representative in showing trends for cases with $n_{u}=1.0-$ $2.0 \times 10^{19} \mathrm{~m}^{-3}$. Profiles of electron density and temperature reach the coupling steady state already at iteration $=1$ although $c_{e}$ near the target, $\gamma_{e} \|$ and $\Delta \phi$ require more iterations.

\subsubsection{Challenge posed by the steep temperature drop}

When the stagnation point density increases up to $2.0 \times 10^{19} \mathrm{~m}^{-3}$ and higher, $c_{e}$ in the near target region converges quickly, however, the upstream parameters (heat conduction coefficient, electron temperature) evolve slowly because of the time step limitation posed by Eq. (24). The large difference between electron collision times upstream and downstream presents a challenge for the kinetic code by requiring large computation times. This provides a motivation for alternative ways of specifying the time step.

Since the initial KIPP run always requires more steps, we increase the time step in the initial KIPP run and then decrease it gradually to satisfy the condition given by Eq. (24) during last iterations. Such a case will be referred to

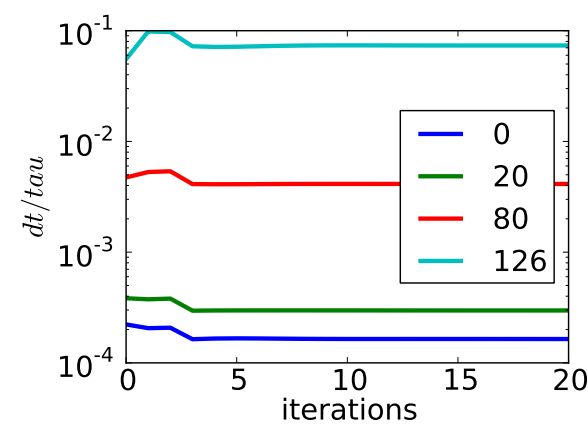

(a)

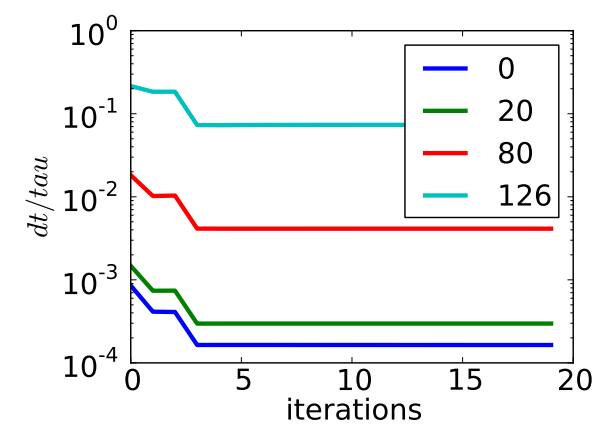

(b)

Figure 13: Evolution of the ratio of the specified time step to the Trubnikov collision time [65] calculated based on parameters at cell 0: the stagnation point, cell 20: upstream, cell 80: downstream, cell 126: the cell adjacent to the target for the (a) "original" and (b) "test" cases. 


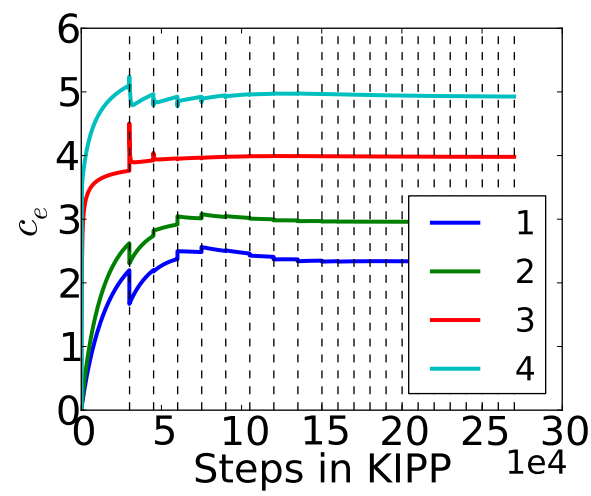

(a) Evolution of $c_{e}$ at the 4 locations within KIPP in the "original" case.

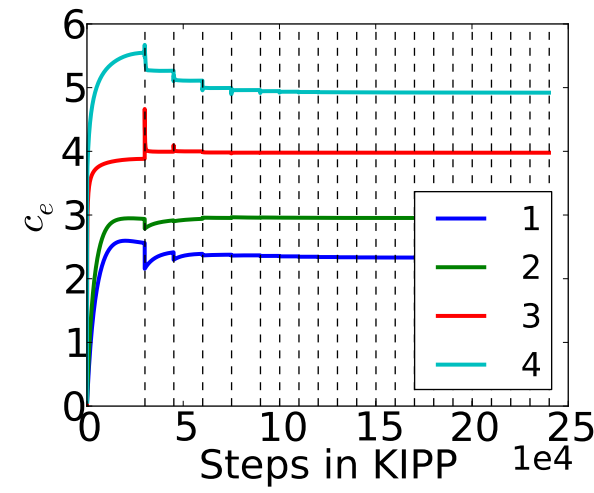

(b) Evolution of $c_{e}$ at the 4 locations within KIPP in the "test" case.

Figure 14: Comparison of the evolution of heat conduction coefficients between two cases (the "original" and "test" cases), with the stagnation point density $n_{u}=2.0 \times 10^{19} \mathrm{~m}^{-3}$.

as the "test" case below, with the standard case, with constant time step $d t$, referred to as the "original" case.

Two cases with $n_{u}=2.0 \times 10^{19} \mathrm{~m}^{-3}$ : "original" and "test" cases, are run and compared below. The only difference between them is the size of time steps

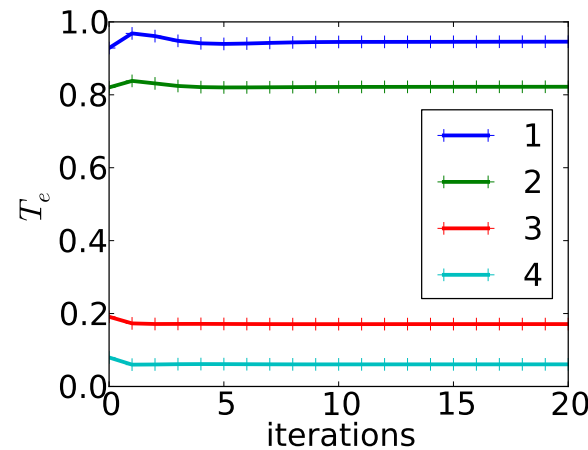

(a) Evolution of $T_{e}$ at the four locations in the "original" case.

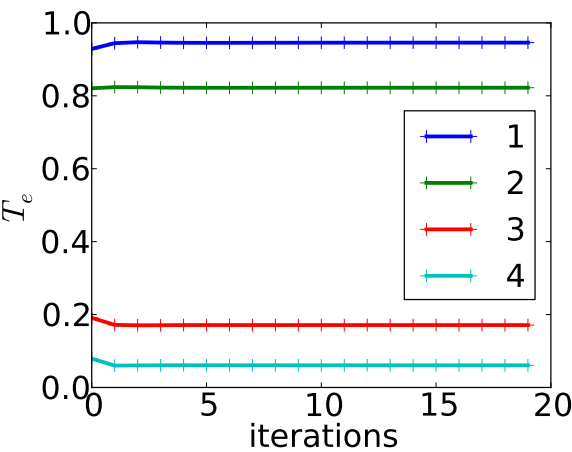

(b) Evolution of $T_{e}$ at the four locations in the "test" case.

Figure 15: Comparison of the evolution of electron temperature profiles between the two cases (the "original" and the "test" cases) with $n_{u}=2.0 \times 10^{19} \mathrm{~m}^{-3}$. 


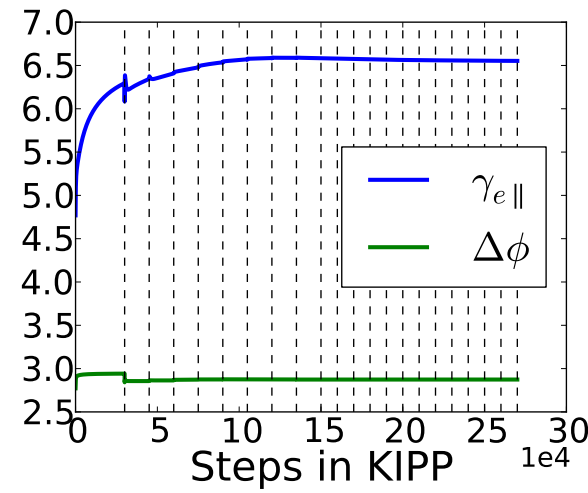

(a) Evolution of $\gamma_{e \|}$ and $\Delta \phi$ within KIPP in the "original" case.

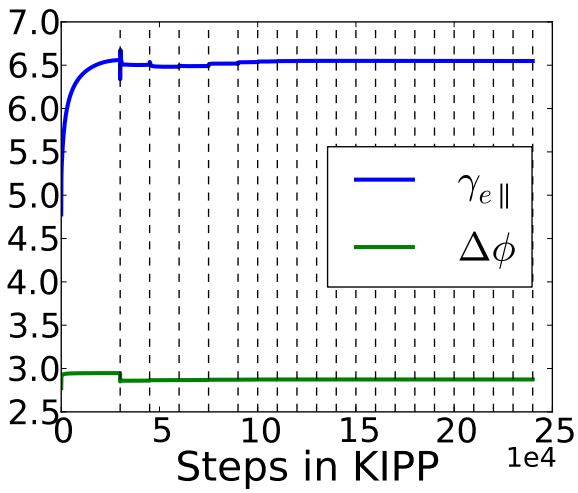

(b) Evolution of $\gamma_{e \|}$ and $\Delta \phi$ within KIPP in the "test" case.

Figure 16: Comparison of the evolution of the electron heat transmission coefficients and sheath potential drops between the two cases (the "original" and the "test" cases) with $n_{u}=$ $2.0 \times 10^{19} \mathrm{~m}^{-3}$.

specified for the initial, second and third runs of KIPP (see Fig. 13 where the ratio of the specified time step to electron collision times calculated at cell 0 : the stagnation point, cell 20: upstream, cell 80: downstream, cell 126: the cell adjacent to the target, is shown for the (a) "original" and (b) "test" cases). The two cases both reach the same coupling steady state, however, it can be clearly seen that in the "test" case, upstream $c_{e}$ (at locations 1 and 2, corresponding to $x \approx 0.890 \mathrm{~m}, x \approx 1.514 \mathrm{~m}$ ) reaches the coupling steady state faster, despite downstream $c_{e}$ (at locations 3 and 4 , corresponding to $x \approx 2.485 \mathrm{~m}, x \approx 2.499 \mathrm{~m}$ ) requires more iterations (compare Figs. 14a and 14b) than the "original" case due to larger time steps specified at the first three KIPP runs. The larger time steps in the "test" case speed up the evolution of upstream temperatures (locations 1 and 2) without impacting the downstream temperatures evolution (locations 3 and 4, see Figs. 15a and 15b). It is surprising that $\gamma_{e \|}$ in the "test" case also converges faster (Figs. 16a and 16b) which is probably attributed to the fact that the electron heat flux transport through the boundary is only determined by the high energy electrons with $v_{\|}>v_{c}$, coming from the upstream 
region. The method adopted in the "test" case can be used for cases with substantial temperature drops.

\subsubsection{High stagnation point density $n_{u}=2.5 \times 10^{19} \mathrm{~m}^{-3}$}

The "test" case method is used here for the case with the stagnation point density $n_{u}=2.5 \times 10^{19} \mathrm{~m}^{-3}$. The upstream collisionality of this case is $\sim 40$, which is quite collisional even for Heat Carrying Electrons (HCE):

$$
\nu_{\mathrm{HCE}}^{*} \approx \frac{40}{25}=1.6>1
$$

The profiles of $n_{e}, T_{e}$ and $\gamma_{e \|}, \Delta \phi$ only require 2 iterations to achieve the coupling convergence. Although this case still shows heat flux limiting upstream and heat flux enhancement downstream (up to factor 1.5 near the target) as shown in Fig. 17, the $T_{e}$ profile in the coupling steady state is quite close to the fluid model result, with only a slight decrease in target electron temperature $(\sim$ $25 \%$, from $1.6 \mathrm{eV}$ to $1.15 \mathrm{eV}$ ) and an increase in $\gamma_{e \|}$ (from 5 to 5.8 ). The electron distribution function $f_{e}$ at the target shows a high energy tail, which however is not so pronounced to significantly impact the value of $\gamma_{e \|}$ (see section 6.1).

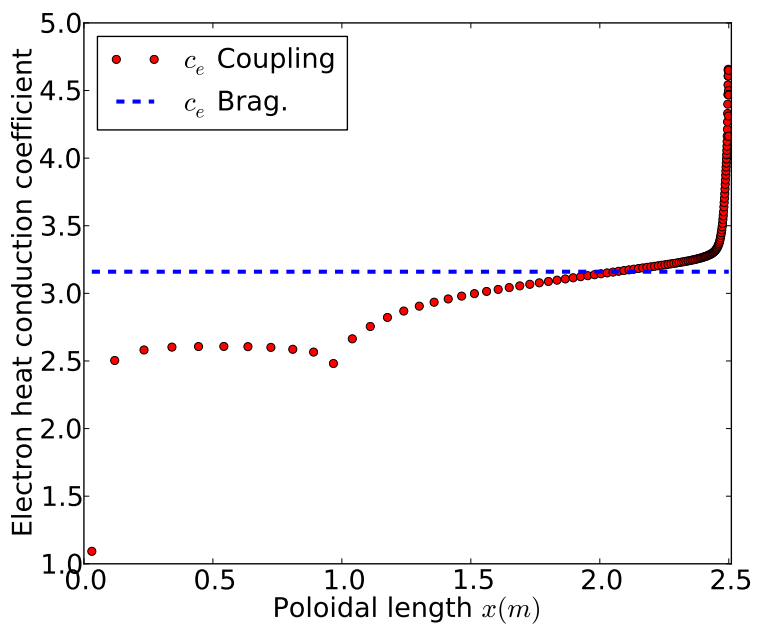

Figure 17: The coupling steady state profile of $c_{e}$ for the case with $n_{u}=2.5 \times 10^{19} \mathrm{~m}^{-3}$. 
Coupling steady state profiles of $n_{e}$ and $T_{e}$ with higher stagnation point density are expected to converge to the fluid ones. However, cases with the electron temperature at the final cell smax smaller than $\sim 1 \mathrm{eV}$ will cause new numerical instability with coupling iterations. The profile of $c_{e}$ will start to oscillate after several coupling iterations for reasons that are not yet clearly understood.

\subsection{Limitations of the iterative coupling algorithm and a possible solution}

The numerical instability of evolving $c_{e}$ in the region near the target reveals the failure of the effort of describing conductive heat flux densities based on local temperature gradients. As shown in section Appendix A, the electron parallel heat flux density in SOLPS consists of two contributions: the conductive heat flux density proportional to the local electron temperature gradient and the convective heat flux density $\frac{5}{2} \Gamma_{e \|} T_{e}$. Up to now, only the conductive piece was manipulated to incorporate the contribution of kinetic effects in a coupling run, which failed in the low upstream collisionality case. Hence a new numerical coupling scheme is proposed and investigated below.

\subsubsection{New scheme}

Since the numerical instability occurs in the region where it shows flux enhancement $\left(c_{e f f}>3.16\right)$ near the target, the main idea of the new scheme is to transfer the enhanced electron heat flux density, captured by KIPP, not only to the conductive heat flux density (by increasing $c_{e}$, causing the numerical instability in low density cases) but also to the convective piece (by increasing the coefficient, which was 2.5 , in the convective heat flux formula). So the electron heat flux density in SOLPS is modified as:

$$
\gamma_{v_{e}} T_{e} \Gamma_{e \|}-c_{e f f} n_{e} \tau_{e} \frac{T_{e}}{m_{e}} \nabla_{\|} T_{e}
$$


The conductive piece (the second term) is still retained because of numerical stability issue discussed later. Initially

$$
\begin{gathered}
\gamma_{v_{e}}=2.5 \\
c_{e f f}=3.16
\end{gathered}
$$

are adopted as a default input. In a coupling run, the enhanced heat flux density calculated in KIPP is split into two parts by determining $\gamma_{v_{e}}$ and $c_{e f f}$ in SOLPS:

$$
\gamma_{v_{e}} T_{e} \Gamma_{e \|}-c_{e f f} n_{e} \tau_{e} \frac{T_{e}}{m_{e}} \nabla_{\|} T_{e}=\frac{1}{2} m_{e} \int f_{e}^{\mathrm{kipp}} v^{2} v_{\|} d \vec{v}
$$

with

$$
\begin{gathered}
\gamma_{v_{e}} \geq \frac{5}{2} \\
c_{e f f} \leq c_{\text {lim }}
\end{gathered}
$$

where $c_{\text {lim }}$ is the user-specified limiting constant determining the splitting ratio of the two parts (i.e. the values of $\gamma_{v_{e}}$ and $c_{e f f}$ ). In later discussions, we will show that the coupling steady state does not depend on the choice of $c_{\text {lim }}$. We define a coefficient $c_{e}^{\prime}$, which is the effective heat conduction coefficient when transferring all the enhanced part to the conductive piece, as:

$$
c_{e}^{\prime}=\left(\frac{1}{2} m_{e} \int f_{e}^{\mathrm{kipp}} v^{2} v_{\|} d \vec{v}-\frac{5}{2} T_{e} \Gamma_{e \|}\right) /\left(-n_{e} \tau_{e} \frac{T_{e}}{m_{e}} \nabla_{\|} T_{e}\right)
$$

Then $c_{e}^{\prime}$ is compared to the limiting coefficient $c_{\text {lim }}$. If $c_{e}^{\prime}$ exceeds the limiting coefficient $c_{\text {lim }}$, we limit the effective heat conduction coefficient to $c_{\text {lim }}$ instead of $c_{e}^{\prime}\left(c_{e f f}=c_{\mathrm{lim}}\right)$, then transfer the further enhanced part to the convective piece (i.e. $\gamma_{v_{e}}$ is increased above 2.5). Otherwise, we transfer all the enhanced flux density to the conductive piece by specifying $c_{e f f}=c_{e}^{\prime}$ and $\gamma_{v_{e}}=\frac{5}{2}$. The analytical expression for determining $\gamma_{v_{e}}$ and $c_{e f f}$ can be found from the following equations:

$$
\begin{aligned}
& \text { if } c_{e}^{\prime} \leq c_{\lim }\left\{\begin{array}{l}
\gamma_{v_{e}}=\frac{5}{2} \\
c_{e f f}=c_{e}^{\prime}
\end{array}\right. \\
& \text { if } c_{e}^{\prime}>c_{\lim }\left\{\begin{array}{l}
\gamma_{v_{e}}=\frac{5}{2}+\left(c_{e}^{\prime}-c_{\lim }\right) \frac{\tau_{e} \nabla_{\|} T_{e}}{m_{e} u_{e} \|} \\
c_{e f f}=c_{\lim }
\end{array}\right.
\end{aligned}
$$




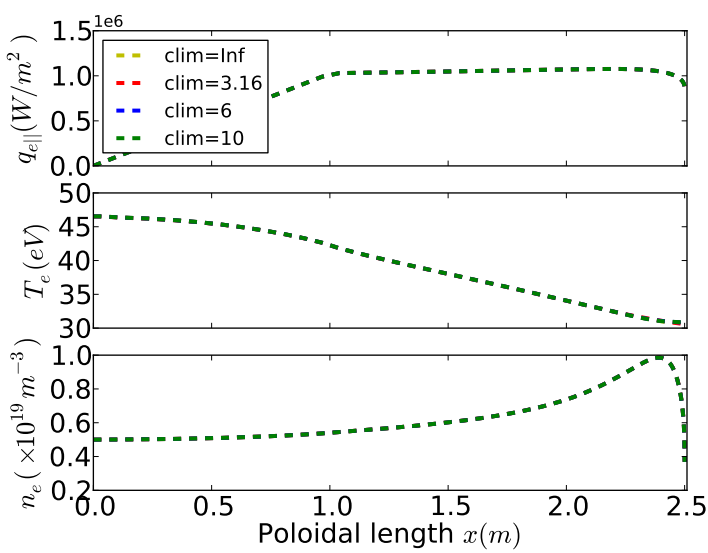

Figure 18: Coupling steady state profiles of $q_{e \|}, T_{e}$ and $n_{e}$ are compared among various limiting coefficients: $c_{\lim }=\infty, c_{\lim }=3.16, c_{\lim }=6, c_{\lim }=10$, for the case with the stagnation point density $0.5 \times 10^{19} \mathrm{~m}^{-3}$.

The conditions $\gamma_{v_{e}}=2.5, c_{e f f}=3.16$ must be satisfied in the collisional limit. In the case of flux limiting (normally in the upstream region), $c_{e f f}$ is reduced, $\gamma_{v_{e}}=2.5$. On the other hand, $c_{e f f}$ is increased in the case of flux enhancement (downstream near the target), however, its increase is limited to the threshold $c_{\lim }$, with any further enhancement moved to the convective piece.

The conductive term in Eq. (31) is still retained since it helps to avoid the numerical instability, although it is pointed out in section 5.4.1 that the formula of conductive heat flux density completely fails in the target region with low upstream collisionality. Completely removing the conductive term was found to cause numerical instabilities in SOLPS. The other kinetic factors: $k_{\|}, \gamma_{e \|}$ and $\Delta \phi$ are still calculated by Eqs. (B.16), (B.33) and (B.21). This coupling scheme will be investigated in the next part of this section.

\subsubsection{Testing the new scheme}

Three limiting coefficients: $c_{\lim }=3.16, c_{\lim }=6, c_{\lim }=10$ are tested for the case with $n_{u}=0.5 \times 10^{19} \mathrm{~m}^{-3}$ and then compared to the one in section 5.4 without the limiting coefficient $\left(c_{\lim }=\infty\right)$. They all achieve the same steady state profiles as shown in Fig. 18. The coupling steady state profiles of $c_{e f f}$ 


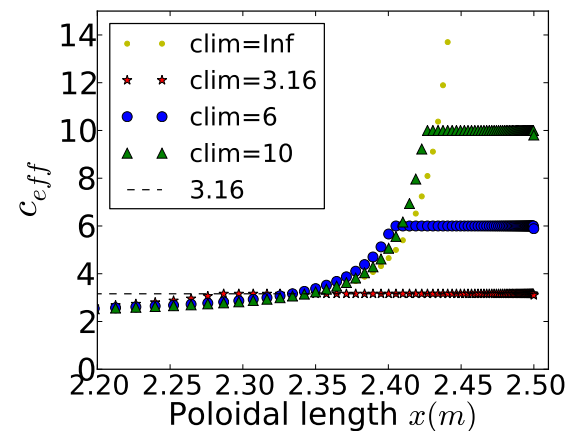

(a)

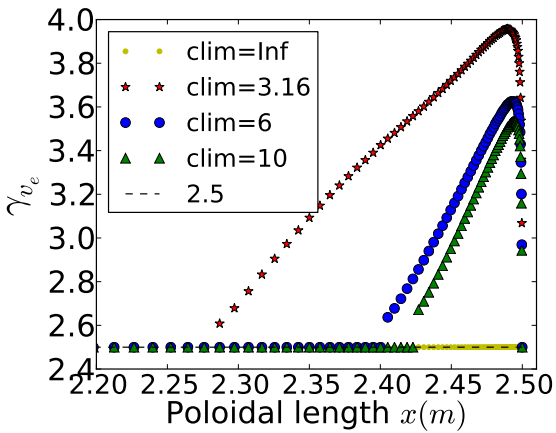

(b)

Figure 19: Coupling steady state profiles of $c_{e f f}$ (a) and $\gamma_{v_{e}}$ (b) for various $c_{\text {lim }}$ with the stagnation point density $n_{u}=0.5 \times 10^{19} \mathrm{~m}^{-3}$.

and $\gamma_{v_{e}}$ are compared among these three cases with various limiting coefficients in Fig. 19, where it can be clearly seen that $c_{e f f}$ is limited to $c_{\lim }$ near the target and $\gamma_{v_{e}}$ in the corresponding region is increased above 2.5. In order to further test the performance of this numerical scheme, $c_{\lim }=3.16$ is also tested for the cases with the stagnation point densities $n_{u}=1.5 \times 10^{19} \mathrm{~m}^{-3}$ and $n_{u}=2.0 \times 10^{19} \mathrm{~m}^{-3}$. As expected, the steady state profiles of $n_{e}, T_{e}$ and $q_{e \|}$ are the same despite a slight increase $(5 \%)$ of $\gamma_{e \|}$ for the case with $n_{u}=2.0 \times 10^{19} \mathrm{~m}$ and with $c_{\mathrm{lim}}=3.16$, resulting in slightly steeper electron temperature profile near the target, which is even unobservable.

Since the new scheme gives the same simulation results as the iterative coupling scheme even for the low collisionality case, later discussions in this work are based on the iterative coupling scheme (corresponding to the new scheme with $\left.c_{\lim }=\infty\right)$.

\section{Discussions}

6.1. Pure D cases

6.1.1. Effects of collisionality 
In this section, $\gamma_{e \|}$ and $\Delta \phi$ in the coupling steady states are compared with the classical values $\gamma_{e \|} \approx 5.0$ and $-e \Delta \phi / T_{e} \approx 3.0$, calculated based on the Maxwellian distribution, for all scanned cases with various collisionalities, as shown in Fig. 20. The sheath potential drop is rather insensitive to

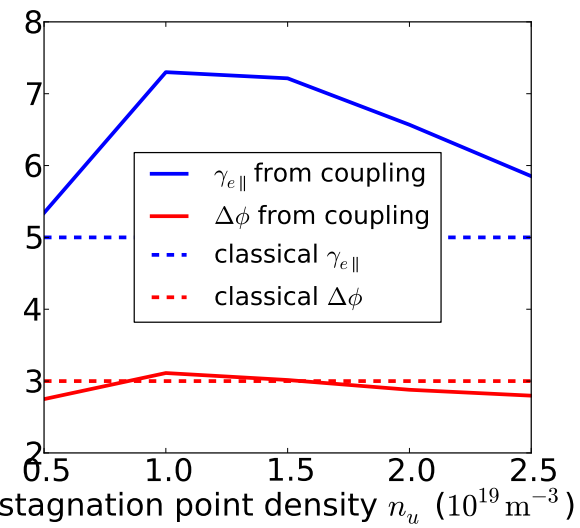
the collisionality variation. The electron heat transmission coeffiFigure 20: The comparison between the kinetic cient is first increasing and then deelectron heat transmission coefficients, the sheath creasing with collisionality $[36,37$, $34,79]$, being close to the classical value for cases with low and high stagnation point densities. However, it can be $\sim 50 \%$ higher than the classical value for cases with medium collisionalities. Accordingly, the extended high energy tail of the distribution function downstream is pronounced at medium upstream collisionalities [32, 35, 37, 33, 59] (see Figs. 21f and 21g), but it is not obvious at low and high collisionalities (see Figs. 21e and 21h) due either to a small temperature drop, so that upstream electrons have similar energies to those downstream, or to the plasma being too collisional causing fast maxwellization of the high energy tail appearing due to electron free-streaming [27].

\subsubsection{Effects of the extended tail on the target temperature}

The coupling steady state 1D distribution functions (defined by Eq. 26) for low $\left(n_{u}=0.5 \times 10^{19} \mathrm{~m}^{-3}\right)$, medium $\left(n_{u}=1.0 \sim 1.5 \times 10^{19} \mathrm{~m}^{-3}\right)$ and high $\left(n_{u}=2.5 \times 10^{19} \mathrm{~m}^{-3}\right)$ stagnation point density cases are shown, for the cell adjacent to the target in Fig. 21: (e), (f), (g), (h), and for cell 10 in Fig. 21: (i), (j), (k), (l). We introduce a 1D heat flux distribution $q_{1 \mathrm{D}}$, defined as a surface 
integral along a certain surface in the velocity space:

$$
q_{1 \mathrm{D}}(\varepsilon)=\frac{1}{2} m_{e} \oint_{v_{\|}^{\prime 2}+v_{\perp}^{2}=2 \varepsilon} f_{e} v^{\prime} v_{\|}^{\prime} d S_{v}
$$

where

$$
\begin{array}{r}
v_{\|}^{\prime}=v_{\|}-u_{e} \\
v^{\prime}=\sqrt{v_{\|}^{\prime 2}+v_{\perp}^{2}}
\end{array}
$$

$u_{e}$ is electron fluid velocity. $S_{v}$ is the surface satisfying $v_{\|}^{\prime 2}+v_{\perp}^{2}=2 \varepsilon$ in the velocity space with $\varepsilon$ being arbitrary kinetic energy. A dimensionless 1D heat flux distribution $\tilde{q}_{1 \mathrm{D}}$ is thus defined as:

$$
\tilde{q}_{1 \mathrm{D}}(\varepsilon)=\frac{q_{1 \mathrm{D}}(\varepsilon)}{q_{1 \mathrm{D}}^{\max }}
$$

where $q_{1 \mathrm{D}}^{\max }$ is the largest value of $q_{1 \mathrm{D}}(\varepsilon)$ with varying $\varepsilon$ in the velocity space. Dimensionless 1D heat flux distributions against kinetic energy $\varepsilon$ (normalized by local electron temperature) are shown in Fig. 21 (red lines denote the ones calculated by the distribution functions at cell 10 and blue lines denote those at the cell adjacent to the target): (a), (b), (c), (d). By relating the extended high energy tails in Fig. 21: (a), (b), (c), (d), with the corresponding values of $\gamma_{e \|}$ for the four cases: $5.3,7.3,7.2,5.8$, it can be concluded that the extended high energy tails are mainly responsible for the increases of the electron heat transmission coefficient compared to the classical value (5.0).

Fig. 22 shows the difference between the target electron temperature $\left(T_{e t}\right)$ in the coupling steady state case and that from the initial coupling iteration (which is the steady state in the SOLPS only case without any kinetic effects) for the scanned cases. For the cases with stagnation point densities $1.0 \times 10^{19} \mathrm{~m}^{-3}$ and $1.5 \times 10^{19} \mathrm{~m}^{-3}$, the coupling steady state $T_{\text {et }}$ drops by $\sim 45 \%$ and $\sim 43 \%$ while the sheath heat transmission coefficient increases to 7.30 (by $\sim 45 \%$ ) and 7.21 (by $\sim 40 \%$ ), respectively, compared to the values without kinetic effects (see Fig. 23). The decrease of $T_{e t}$ due to kinetic effects seems to be related mostly to the deviation of the electron heat transmission coefficients from the classical values. This is discussed further in [75, 95]. 

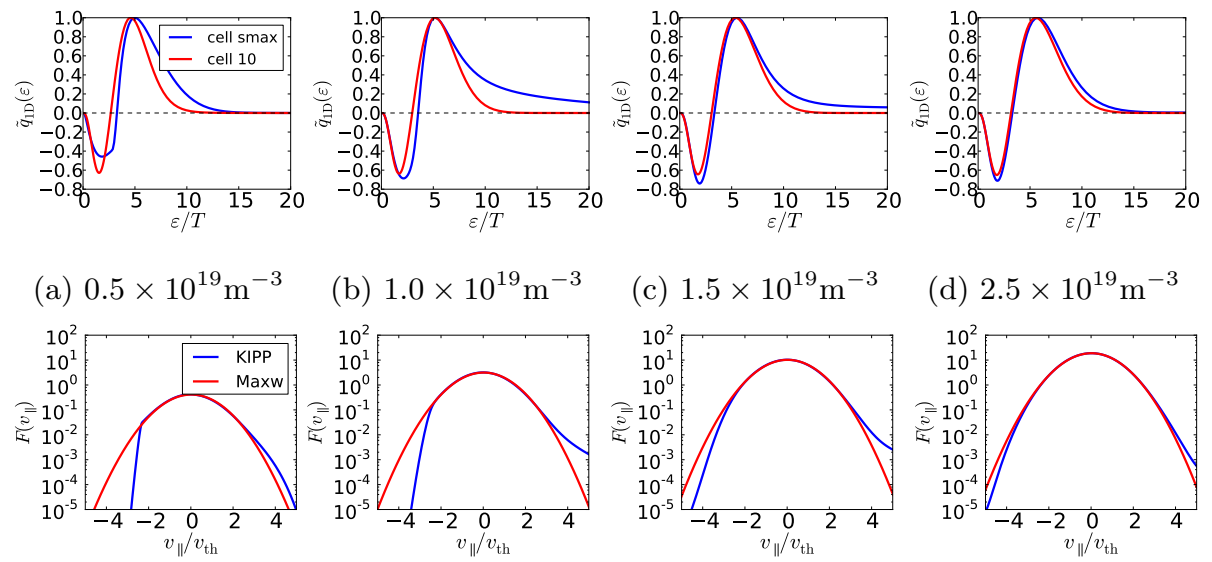

(b) $1.0 \times 10^{19} \mathrm{~m}^{-3}$

(c) $1.5 \times 10^{19} \mathrm{~m}^{-3}$

(d) $2.5 \times 10^{19} \mathrm{~m}^{-3}$

(e) $0.5 \times 10^{19} \mathrm{~m}^{-3}$
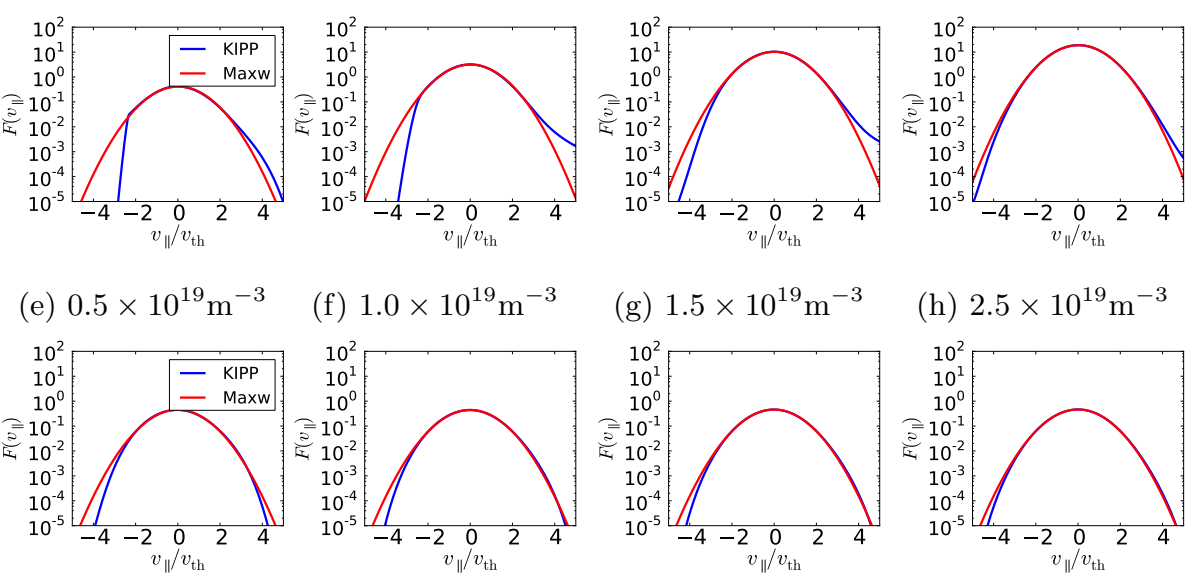

(f) $1.0 \times 10^{19} \mathrm{~m}^{-3}$

(g) $1.5 \times 10^{19} \mathrm{~m}^{-3}$

(h) $2.5 \times 10^{19} \mathrm{~m}^{-3}$

(i) $0.5 \times 10^{19} \mathrm{~m}^{-3}$
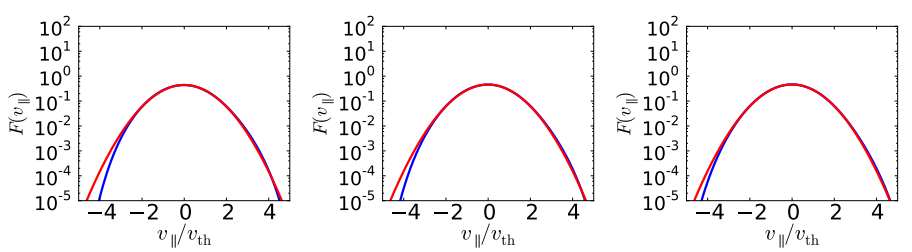
(j) $1.0 \times 10^{19} \mathrm{~m}^{-3}$

(k) $1.5 \times 10^{19} \mathrm{~m}^{-3}$

(l) $2.5 \times 10^{19} \mathrm{~m}^{-3}$

Figure 21: Dimensionless 1D heat flux distribution at the cell (cell smax) next to the target (in blue) and at cell 10 corresponding to the position at $\sim \frac{1}{3}$ of the simulation domain upstream (in red) are shown for a) $n_{u}=0.5 \times 10^{19} \mathrm{~m}^{-3}$, b) $n_{u}=1.0 \times 10^{19} \mathrm{~m}^{-3}$, c) $n_{u}=1.5 \times 10^{19} \mathrm{~m}^{-3}$, d) $n_{u}=2.5 \times 10^{19} \mathrm{~m}^{-3}$. The corresponding $1 \mathrm{D}$ distribution functions against $v_{\|}$are shown at cell $\max$ (in blue) in e,f,g,h and those at cell 10 are shown (in blue) in i,j,k,l, the red curves in e,f,g,h,i,j,k,l are local Maxwellian distribution functions.

\section{2. $D+C$ case}

From the above discussions, it can be concluded that kinetic effects of electron parallel transport are primarily attributed to two factors [32]:

1. Non-local effects of high energy downstreaming electrons featured by the extended high energy $f_{e}$ tail towards the target in medium or even highly collisional cases.

2. Asymmetric target sink characterized by a cut-off $f_{e}$ in the region near the target in weakly collisional cases. 


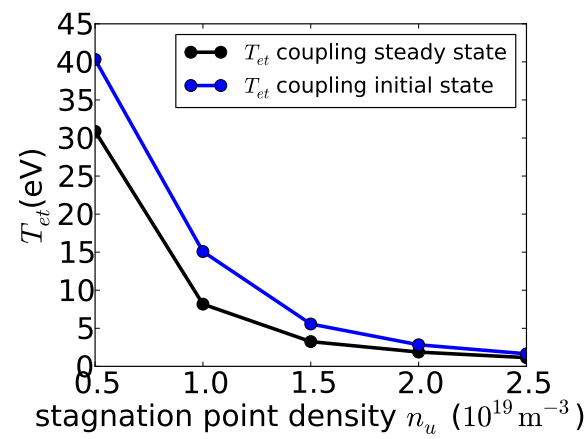

Figure 22: Target electron temperature of a coupling steady state (black) and of a SOLPS steady state (blue) for various cases with different stagnation point densities.

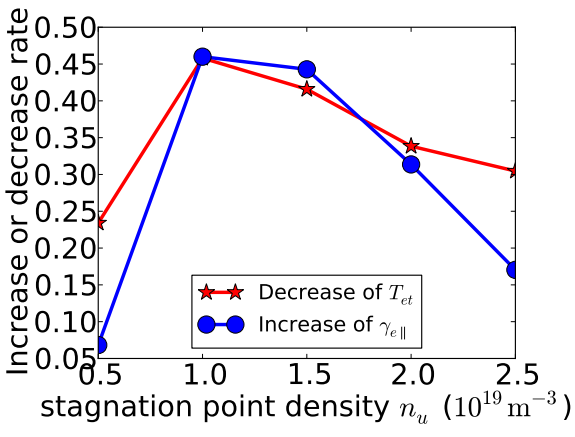

Figure 23: The degree of the decrease in the target $T e$ and the increase in $\gamma_{e} \|$ compared to the SOLPS results (without coupling with KIPP) for various coupling cases with different $n_{u}$.

There are two key conditions that determine the importance of the non-local effects of high energy downstreaming electrons:

- Upstream collisionality $\nu^{*}=\frac{L_{p a r}}{\lambda_{u}}$.

- Electron temperature drop $T_{e u} / T_{e t}$

Medium upstream collisionality cases with pure deuterium plasmas already show observable kinetic effects. In order to achieve stronger electron temperature drops but with unchanged upstream collisionalities, carbon, used as a radiator, is introduced in the coupling simulation as an impurity.

\subsubsection{Simulation setup}

The upstream deuterium ion density is set at $2.2 \times 10^{19} \mathrm{~m}^{-3}$, and power input is set to match the power flux through the separatrix with constant flux density $0.132 \mathrm{MW} / \mathrm{m}^{2}$ from the stagnation point to $\sim \frac{1}{4}$ of the simulation domain (corresponding to the first 7 cells, from $x=0 \mathrm{~m}$ to $x \approx 0.68 \mathrm{~m}$ ). The cells with power input are hence separated from the region where the radiation power is concentrated (downstream). Chemical and physical impurity sputtering models in SOLPS are activated to produce carbon impurities. The constant sputtering 
yield with deuterium sputtering coefficient 0.095 is prescribed for the chemical sputtering model. TRIM database is used for the physical sputtering model. The recycling coefficient of carbon particles (including all charged states and neutrals) is set to zero while the deuterium recycling coefficient is set to 0.999 .

One problem for the 1D geometry with carbon impurity was that carbon ions, especially highly charged ions (particularly $C^{4+}$ ), tended to concentrate upstream due to thermal force since there were no sinks for them. This concentration would make the evolution unstable and cause the cases to crash even if the chemical sputtering coefficient is prescribed at a very small value. To avoid this problem, an artificial carbon ion particle sink is introduced along the simulation domain, mimicking the radial loss out of a 2D geometry. The sink loss rate is specified as:

$$
S_{C^{i+}}=-r_{i} n_{C^{i+}} \sqrt{\frac{T_{i}}{m_{i}}} \quad i=0,6
$$

where $n_{C^{i+}}$ is particle density of species $C^{i+}$. Carbon neutrals have $i=0$, and $S_{C^{i+}}$ is particle loss rate of species $C^{i+} . r_{i}$ is the loss rate coefficient, a free dimensionless parameter to be prescribed by the user. In this case, in order to efficiently remove highly charged ions upstream, the loss rate coefficient $r_{i}$ is prescribed to be proportional to the local pressure of $C^{i+}$, as:

$$
\begin{array}{rlrl}
r_{i} & =\frac{n_{C^{i+}}(x) T_{i}(x) L_{p o l}}{\int n_{C^{i+}}(x) T_{i}(x) d x} & i & =3,6 \\
r_{i} & =0 & i & =0,2
\end{array}
$$

where $L_{p o l}$ is poloidal length of the simulation domain and $x$ is poloidal coordinate.

\subsubsection{Coupling test with carbon impurity}

The upstream collisionality is $\sim 18$, comparable to the case with $n_{u}=$ $1.0 \times 10^{19} \mathrm{~m}^{-3}$ of pure deuterium plasmas (section 5.4), however, the electron temperature drop $T_{e u} / T_{e t} \approx 12.20$ here is larger by about factor 2 . Such a medium upstream collisionality, but with the substantial electron temperature drop, results in an extended high energy tail of the 1D distribution function 


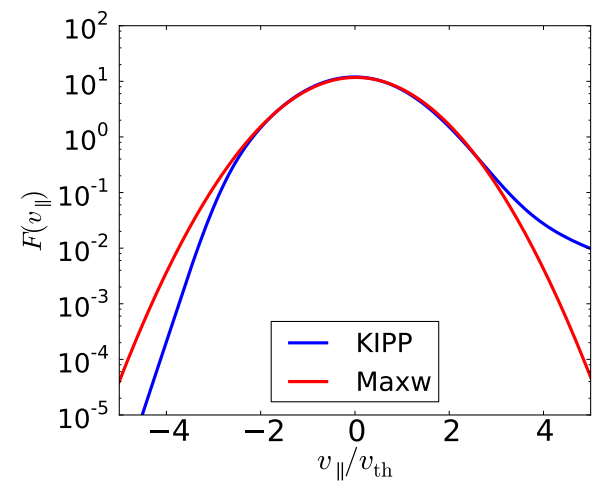

(a) Cell next to the target.

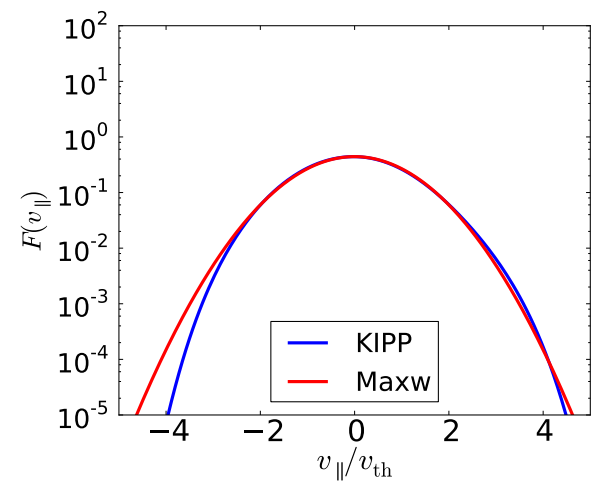

(b) Cell 10 .

Figure 24: 1D distribution functions (Eq. (26)) at the cell adjacent to the target (a) and the cell 10 (b). Blue curves denote the distribution function calculated by KIPP while red curves denote local Maxwellian.

at the cell adjacent to the target, as shown in Fig. 24a. The contour plot of the electron conductive heat flux density and dimensionless 1D heat flux distribution at the same cell are shown in Fig. 25. It can be clearly seen that the contribution of high energy electrons is increased dramatically because of their non-local transport since they experience significantly fewer collisions when moving from upstream to downstream.

The extended high energy tail leads to a rather high electron sheath heat transmission coefficient $\gamma_{e \|} \approx 11.20$, more than $100 \%$ increase from the classical value, while it does not contribute much to the sheath potential drop, $\Delta \phi \sim$ $3.15 \frac{T_{e}}{e}$. Accordingly, the target electron temperature is decreased by $\sim 60 \%$, while the stagnation electron temperature is not much affected, indicating that the kinetic boundary conditions are important in the modification of the profile of electron temperature. More discussions can be found in [75, 95].

\section{Summary}

SOLPS is the main tool for the prediction of SOL and divertor conditions in the future fusion device ITER, where parallel kinetic effects in the SOL 


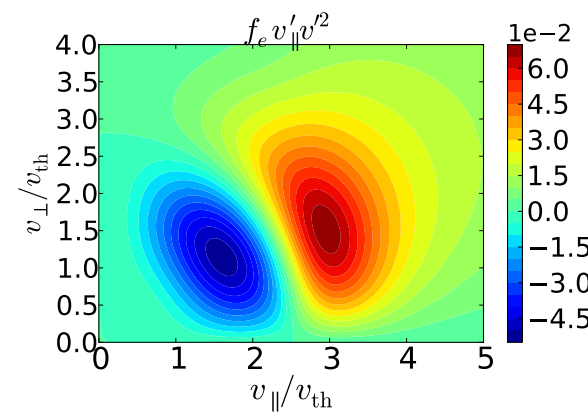

(a) Contour plot of the electron conductive heat flux density in velocity space at the cell adjacent to the target.

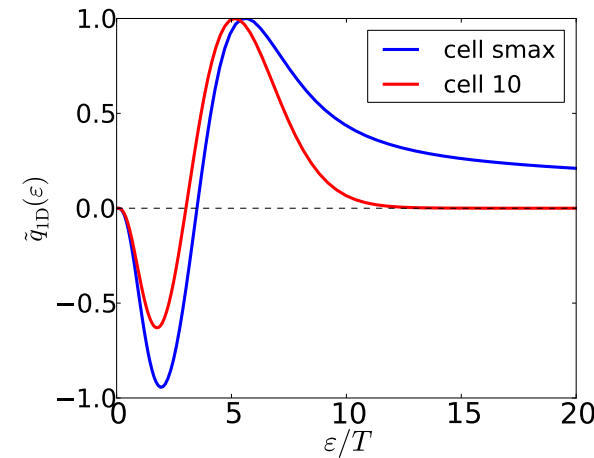

(b) Dimensionless 1D heat flux distribution against kinetic energy $\varepsilon$ (normalized by local electron temperature) at the cell adjacent to the target (blue line) and at cell number number 10 (red line).

Figure 25: 2D and 1D electron conductive heat flux contribution from different velocity.

will play an important role. The present work has enabled SOLPS in its 1D version to incorporate kinetic effects of parallel electron transport by coupling it with KIPP. Extensions of the coupling scheme to a 2D geometry can be simply achieved by regarding the $2 \mathrm{D}$ geometry as a pile-up of the $1 \mathrm{D}$ flux tube in the radial direction.

The coupling algorithm takes advantage of the strong points of SOLPS which solves a highly sophisticated fluid model, with self-consistent recycling and physical and chemical sputtering models, as well as atomic physics, while treating electrons kinetically in order to consider the most important kinetic effects of parallel electron transport. It has been demonstrated that typically only 2 or 3 iterations between SOLPS and KIPP are necessary to achieve a coupling steady state (see section 5.4).

The three coupling schemes: 'center' (Scheme A), 'face'(Scheme B), 'decouple'(Scheme C) give the same steady state profiles (see section 5.3) and Scheme B is suggested for future simulations.

The coupling steady state does not depend on initial conditions, however, 
the convergence efficiency is quite sensitive to the initial profile (see section 5.3). The initial setup with the default coefficients: $c_{e}=3.16, k_{\|}=0.71$ and $\gamma_{e \|}=5$ is suggested.

For cases with the large electron temperature drop $\left(T_{u} / T_{t}>10\right)$, the necessary condition (Eq. (24)) limits the time step to a significantly smaller value compared to the upstream collision time $\tau_{u}$ :

$$
d t<0.1 \tau_{t}<6 \times 10^{-4} \tau_{u}
$$

which leads to slow evolution of upstream parameters. Instead of specifying $d t<0.1 \tau_{t}$ for the entire coupling run, we tested the scheme with varying time step $d t$ from $\sim \tau_{t}$ to $0.1 \tau_{t}$ at the first one or two KIPP runs, which significantly accelerated the evolution of upstream parameters without influencing the downstream parameters evolution (see section 5.4).

One may be suspicious of the idea that the information about non-local transport modelled by KIPP can be wrapped up into a local effective heat conduction coefficient $c_{\text {eff }}$ in the coupling scheme. However, based on the analysis in section 4 , achieving the coupling steady state of the iterative coupling scheme already means treating electrons fully kinetically. The coupling steady state profiles can always be achieved, and they seem to be unique despite the numerical instability of the evolution of heat conduction coefficients (however the heat flux density is already in the steady state since $c_{e f f} \nabla_{\|} T_{e} \approx$ const with coupling iterations) in the region near the target in cases with low upstream collisionality.

The numerical instability appearing in low collisionality cases can be avoided by applying a limiting coefficient $c_{\lim }$ to conductive heat flux density (see sec-

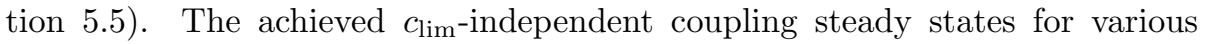
collisionalities imply that the coupling scheme allows one to obtain the unique solution with the kinetic treatment of electrons by simply maintaining profiles of electron density, temperature and particle flux density in KIPP and then transferring kinetic coefficients from KIPP back to SOLPS.

Similar to fully kinetic simulations, the iterative coupling algorithm self- 
consistently achieves the profiles of electron temperature and heat flux density determined by sources, but with the help of the fluid model to provide information that cannot be obtained with only electron kinetic equation. The effective kinetic factors $c_{e f f}, k_{e f f}, \gamma_{e f f}, \Delta \phi_{e f f}$ connect the kinetic electron equation and the fluid equations in the coupling scheme. For example, $c_{e f f}$ acts as a free parameter, matching the relation between electron temperature and heat flux density that is used in the fluid model. It indicates that the specific form of the equation for the relation between electron temperature and heat flux density (the closure equation B.4) doesn't influence the simulation results with the iterative coupling scheme, which only acts as a bridge to transfer information from KIPP to SOLPS (as has been elucidated in section 5.5). The achievement of the coupling steady state is the key. However, the choice of the closure equation should not introduce numerical instabilities, e.g. removing the conductive piece from the closure equation would introduce numerical instability to SOLPS, and hence the coupling steady state would never be reached (see section 5.5).

As was expected (see e.g. [27]), density scan cases with pure deuterium plasmas showed strong kinetic effects in cases with medium upstream collisionalities. However, in real situations, radiation sinks due to impurities are always present in the divertor region. Hence the upstream collisionality $\nu^{*}$ is not the only free parameter to determine the role of kinetic effects. The $T_{e}$ drop is also important. In order to simulate the case with low upstream collisionality but a strong temperature drop which is expected in ITER, we switched on carbon impurity sputtering. Carbon was introduced as a radiator in the coupling simulation. One problem for $1 \mathrm{D}$ geometry with the carbon impurity is that highly charged ions tend to concentrate near the stagnation point, which causes cases to crash. The artificial carbon ion particle sink (see section 6.2) was applied to avoid this problem. The case with the carbon impurity clearly showed stronger kinetic effects. This can be concluded by comparing the case with the carbon impurity and the case with $n_{u}=1.0 \times 10^{19} \mathrm{~m}^{-3}$ with pure deuterium, which have similar upstream collisionalities $\nu^{*} \approx 16-18$ but different electron temperature drops. 


\section{Acknowledgement}

This work has been carried out within the framework of the EUROfusion Consortium and has received funding from the Euratom research and training programme 2014-2018 under grant agreement No 633053. The views and opinions expressed herein do not necessarily reflect those of the European Commission.

\section{Appendix A. 1D SOLPS adaptation}

SOLPS was created as a 2D code, with poloidal and radial directions based on the assumption that all parameters in the toroidal direction are constant. For a number of applications, a 1D version of SOLPS was created [76, 77]. The kinetic code KIPP is mainly aimed at coupling parallel kinetic transport with SOLPS, therefore it is the 1D SOLPS version without currents and drifts, with variables only varying in the poloidal direction, that is used for testing the coupling algorithm (described in section 4) since parallel kinetic effects have no direct interaction with the radial transport.

Fig. A.26 shows the 1D grid cells generated along the poloidal coordinate $(x)$ in SOLPS. Only one cell is created in the radial direction. The radial transport (e.g. ion and electron radial particle flux densities $\Gamma_{i y}, \Gamma_{e y}$ or radial heat flux densities $q_{i y}$ or $q_{e y}$ ) is disabled by switching off drifts and forcing radial gradients to $0\left(\nabla_{y}=0\right)$. Hence radial particle and heat flux densities in this version of SOLPS through the south and north faces of cell $x$ (see Fig. A.26) are

$$
\begin{aligned}
\Gamma_{y}(x) & =0 \\
q_{y}(x) & =0
\end{aligned}
$$

Without the radial transport it becomes essentially a $1 \mathrm{D}$ problem in the poloidal direction, but, as discussed above, the poloidal transport is the sum of projections of parallel transport along the magnetic field line and the transport in the perpendicular direction in the magnetic flux surface on the poloidal 


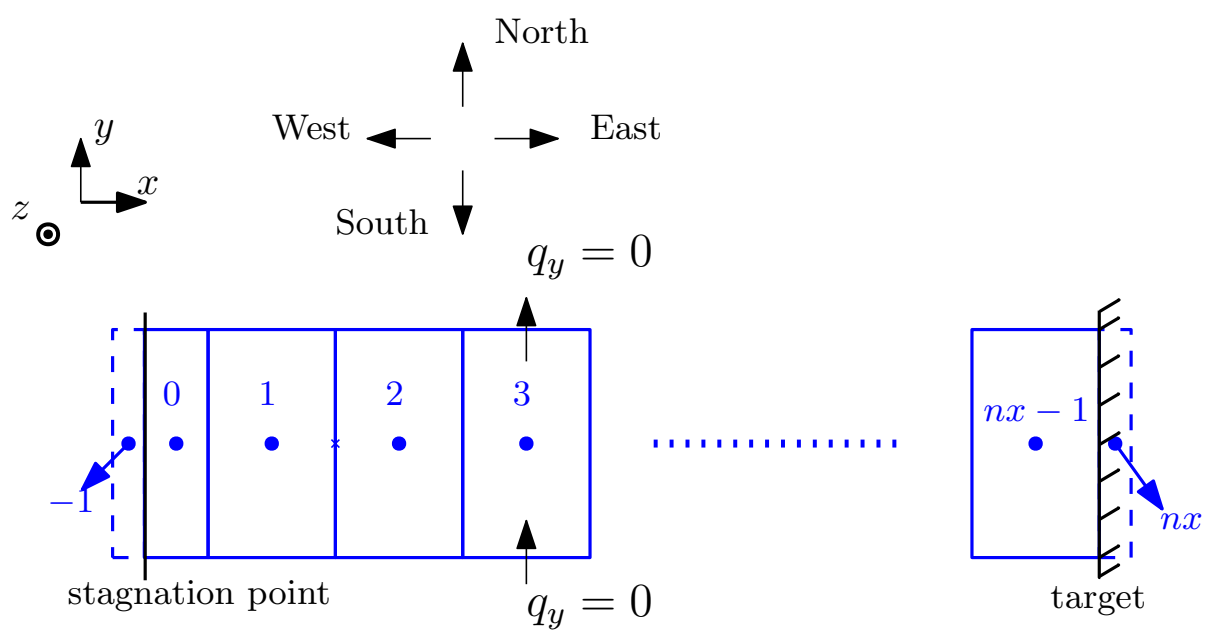

Figure A.26: 1D grid for SOLPS is generated along the poloidal direction $(x)$. Radial particle and heat flux densities (e.g. heat flux densities through north and south cell faces $q_{y}=0$ for all species) are switched off (details can be found in the text). The number of cells is $n x+2$, with two guard cells: cell -1 and $n x$, attached to the west and east boundaries.

direction (see Fig. A.27):

$$
\begin{aligned}
\Gamma_{x}(x) & =b_{x} \Gamma_{\|}(x)+b_{z} \Gamma_{\perp}(x) \\
q_{x}(x) & =b_{x} q_{\|}(x)+b_{z} q_{\perp}(x)
\end{aligned}
$$

where

$$
\begin{aligned}
& b_{x}=\frac{B_{x}}{\sqrt{B_{x}^{2}+B_{z}^{2}}} \\
& b_{z}=\frac{B_{z}}{\sqrt{B_{x}^{2}+B_{z}^{2}}}
\end{aligned}
$$

We assume that the magnetic field is constant, $\vec{B}=$ const, in this work.

Since the anomalous diffusion coefficient $D^{n}$ and electron and ion thermal conductivities $\chi_{e \perp}, \chi_{i \perp}$ are much smaller than the parallel ones, the perpendicular terms are not important. They are regarded as source terms in KIPP (see discussions in section 4).

Hence the adaptation of the Braginskii equations to the 1D geometry results 

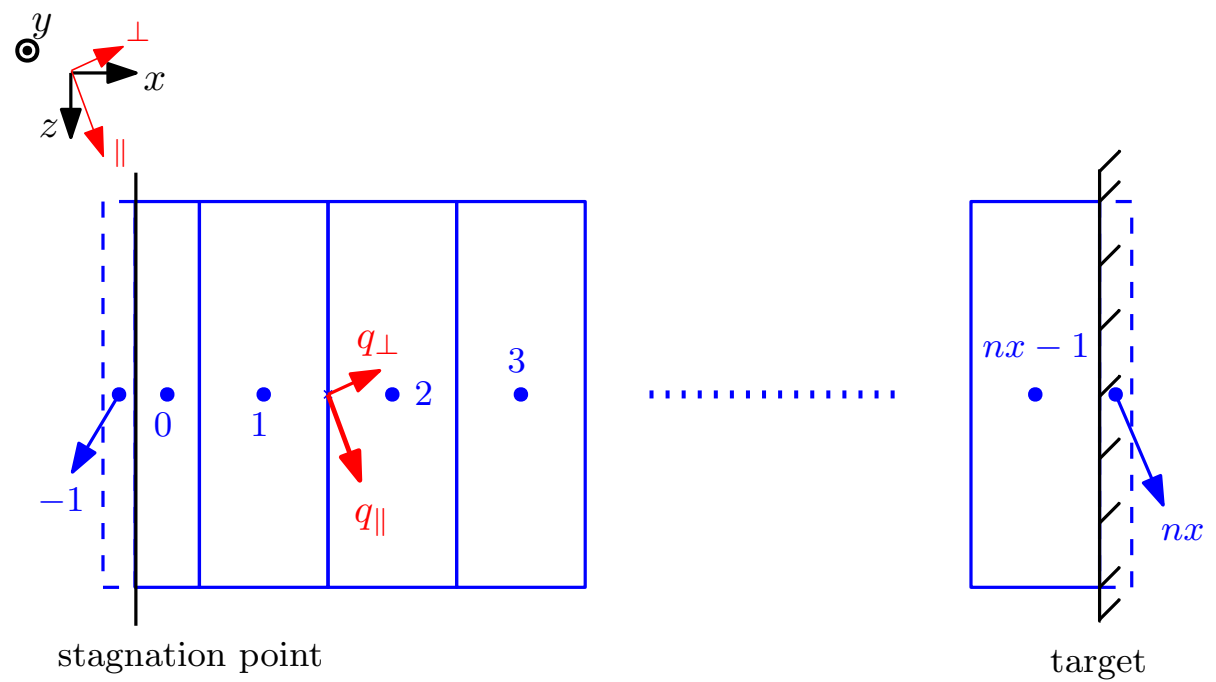

stagnation point

target

Figure A.27: 1D grid for SOLPS (the top view of the grid in Fig. A.26). 1D transport (e.g. $q_{x}$ ) along the poloidal direction $(x)$ at the left face of a certain cell (e.g. cell 2) is the sum of projections of the transport in parallel and perpendicular directions.

in:

$$
\begin{aligned}
& \frac{\partial n_{i}}{\partial t}+\nabla_{x} \Gamma_{i x}=S_{n_{i}} \\
& n_{e}=\sum_{i} Z_{i} n_{i} \\
& \nabla_{x} j_{x}=0 \\
& \frac{\partial}{\partial t}\left(m_{i} n_{i} u_{i \|}\right)+\nabla \cdot\left(m_{i} n_{i} u_{i \|} \vec{u}_{i}\right)=-\nabla_{\|}\left(p_{i}+p_{e}\right)-\nabla \cdot \vec{\Pi}_{i}^{\|}+S_{i \|} \\
& \frac{\partial}{\partial t}\left(\frac{3}{2} n_{i} T_{i}\right)+\nabla_{x}\left(F_{i x} T_{i}+q_{i x}^{\text {cond }}\right)-Q_{\Delta}=S_{E i}-n_{i} T_{i} \nabla_{\|} u_{i \|}-\left(\hat{\Pi}_{i} \cdot \nabla\right) \cdot \vec{u}_{i} \\
& \frac{\partial}{\partial t}\left(\frac{3}{2} n_{e} T_{e}\right)+\nabla_{x}\left(F_{e x} T_{e}+q_{e x}^{\text {cond }}\right)+Q_{\Delta}=S_{E e}-n_{e} T_{e} \nabla_{\|} u_{e \|}+\frac{1}{e n_{e}} j_{x} R_{e x}
\end{aligned}
$$

where $m, n, u, T, p$ are mass, density, velocity, temperature, pressure; $\Gamma, q, j$ are particle, heat flux densities and current density. The subscripts $e$ or $i$ denote the variable for electrons or ions. The subscript \| denotes the vector variable in the parallel direction while the subscript $x$ denotes the sum of projections 
of the vector variable both in parallel and perpendicular directions on the $x$ direction, similar to Eqs. (A.3) and (A.4). $S_{n}$ and $S_{E}$ are particle and energy source terms while $S_{i \|}$ is the ion momentum source term. $R_{e x}$ is the projection of the thermal force on the poloidal direction.

The poloidal electron (or ion) heat flux density includes the convective piece:

$$
q_{e x}^{\mathrm{conv}}=F_{e x} T_{e}
$$

where $F_{e x}$ is poloidal electron particle flux density multiplied by a coefficient:

$$
\begin{aligned}
F_{e x} & =b_{x} F_{e \|}+b_{z} F_{e \perp} \\
F_{e \|} & =\frac{3}{2} \Gamma_{e \|} \\
F_{e \perp} & =\frac{3}{2} \Gamma_{e \perp}^{\mathrm{ExB}}+\frac{5}{2} \Gamma_{e \perp}^{\mathrm{dia}}+\frac{5}{2} \Gamma_{e \perp}^{\mathrm{a}}
\end{aligned}
$$

and the conductive piece:

$$
\begin{aligned}
& q_{e x}^{\text {cond }}=b_{x} q_{e \|}^{\text {cond }}+b_{z} q_{e \perp}^{\text {cond }} \\
& q_{e \|}^{\text {cond }}=-c_{e} \frac{n_{e} T_{e} \tau_{e}}{m_{e}} \nabla_{\|} T_{e} \\
& q_{e \perp}^{\text {cond }}=-\chi_{e \perp} n_{e} \nabla_{\perp} T_{e}
\end{aligned}
$$

where $c_{e}=3.16$ for $Z_{i}=1$ and $\chi_{e \perp}$ is the anomalous thermal conductivity, which is a free parameter specified by the user. $\tau_{e}$ is the electron collision time.

\section{Appendix B. Numerical details of the implementation of the coupling} scheme

Appendix B.1. Comparison between electron balance equations in KIPP and SOLPS

The main goal of the iterative scheme is to incorporate kinetic effects of parallel electron transport calculated by KIPP into SOLPS. Equations solved in SOLPS and KIPP are compared below. 


\section{Appendix B.1.1. Equations solved for electrons in SOLPS}

Since KIPP is only tackling electron parallel transport, while, as discussed in section Appendix A, 1d SOLPS transport is essentially the sum of poloidal projections of parallel and perpendicular transport, the particle and heat flux densities calculated based on $f_{e}$ in KIPP here correspond to parallel parts in Eqs. (A.3) and (A.4). The electron equations (Eqs. (A.8), (A.9) and (A.12)) solved in the SOLPS 1D geometry are shown again here with moving the perpendicular terms to the right hand side and replacing $\nabla_{x}$ terms with $\nabla_{\|}$and $\nabla_{\perp}$ terms:

$$
\begin{gathered}
\frac{\partial n_{e}}{\partial t}+\nabla_{\|}\left(\Gamma_{e \|}\right)=S_{p}-\nabla_{\perp} \Gamma_{e \perp} \\
e n_{e} E_{\|}=R_{T_{\|}}-\nabla_{\|} p_{e} \\
\frac{\partial}{\partial t}\left(\frac{3}{2} n_{e} T_{e}\right)+\nabla_{\|}\left(\frac{3}{2} n_{e} T_{e} u_{e \|}+q_{e \|}^{\text {cond }}\right) \\
=-n_{e} T_{e} \nabla_{\|} u_{e \|}-Q_{\Delta}+S_{E e}-\nabla_{\perp} q_{e \perp}
\end{gathered}
$$

and the closure equations:

$$
\begin{aligned}
q_{e \|}^{\text {cond }} & =-c_{e} n_{e} \tau_{e} \frac{T_{e}}{m_{e}} \nabla_{\|} T_{e} \\
R_{T_{\|}} & =-k_{\|} n_{e} \nabla_{\|} T_{e}
\end{aligned}
$$

where $c_{e}=3.16, k_{\|}=0.71$ (default values for all species under the choice of "Braginskii" model in SOLPS). $S_{p}$ and $S_{E e}$ are volumetric particle and energy sources, $E_{\|}$is the electric field, and Eq. (B.2) gives the profile of electric potential.

Appendix B.1.2. Equations solved for electrons in KIPP

The main equation to be solved in KIPP is the Vlasov-Fokker-Planck equation for electron parallel transport shown again here (not dimensionless):

$$
\frac{\partial f_{e}^{\mathrm{k}}}{\partial t}+v_{\|} \nabla_{\|} f_{e}^{\mathrm{k}}-\frac{e E_{\|}}{m_{e}} \frac{\partial f_{e}^{\mathrm{k}}}{\partial v_{\|}}=\left(\frac{\partial f^{\mathrm{k}}}{\partial t}\right)_{\text {coll. }}+S_{\text {energy }}+S_{\text {particle }}
$$

where $f_{e}^{\mathrm{k}}\left(v_{\|}, v_{\mid}, s\right)$ is the $3 \mathrm{D}$ electron distribution function: $2 \mathrm{D}$ in velocity space and $1 \mathrm{D}$ in real space along the magnetic field. KIPP can be easily adapted to 
evolving $f_{e}^{\mathrm{k}}$ while maintaining fixed density and temperature profiles by specifying automatic energy and particle sources. The first three moment equations following from Eq. (B.6) in KIPP are:

$$
\begin{aligned}
& \frac{\partial n_{e}^{\mathrm{k}}}{\partial t}+\nabla_{\|}\left(\Gamma_{e \|}^{\mathrm{k}}\right)=S_{p}^{\mathrm{k}} \\
& e n_{e}^{\mathrm{k}} E_{\|}^{\mathrm{k}}=R_{T_{\|}^{\mathrm{k}}}-\nabla_{\|} p_{e}^{\mathrm{k}} \\
& \frac{\partial}{\partial t}\left(\frac{3}{2} n_{e}^{\mathrm{k}} T_{e}^{\mathrm{k}}\right)+\nabla_{\|} q_{e \|}^{\mathrm{k}}=-e n_{e}^{\mathrm{k}} u_{e \|}^{\mathrm{k}} E_{\|}^{\mathrm{k}}+Q_{c}^{\mathrm{k}}+S_{E e}^{\mathrm{k}}
\end{aligned}
$$

where all variables with the superscript $\mathrm{k}$ are calculated based on the distribution function in KIPP, $f_{e}^{\mathrm{k}}$. The thermal force and electron parallel heat flux density are defined as:

$$
\begin{aligned}
R_{T_{\|}}^{\mathrm{k}} & =\int m_{e} v^{\prime}\left(\frac{\partial f^{\mathrm{k}}}{\partial t}\right)_{\text {coll. }} d \vec{v} \\
q_{e \|}^{\mathrm{k}} & =\frac{1}{2} m_{e} \int f_{e}^{\mathrm{k}} v^{2} v_{\|} d \vec{v}
\end{aligned}
$$

Appendix B.2. Transferring electron profiles from SOLPS to KIPP

As pointed out above in the Step 3 of the coupling scheme, profiles of electron density, velocity and temperature etc. are transferred from SOLPS to KIPP and maintained there:

$$
\begin{gathered}
n_{e}^{\mathrm{k}}=n_{e} \\
u_{e \|}^{\mathrm{k}}=u_{e \|} \\
T_{e}^{\mathrm{k}}=T_{e} \\
\Gamma_{e \|}^{\mathrm{k}}=\Gamma_{e \|}
\end{gathered}
$$

by adjusting $S_{p}^{\mathrm{k}}$ and $S_{E e}^{\mathrm{k}}$.

Appendix B.3. Modifications of kinetic factors in KIPP

Appendix B.3.1. Effective thermal force coefficient $k_{\text {eff }}$

Eqs. (B.7) and (B.8) are consistent with Eqs. (B.1) and (B.2) which are solved in SOLPS. The profile of effective thermal force coefficient can be obtained at the end of each KIPP loop (the quasi-steady state is supposed to be 
achieved, details can be found in section 5.2). This coefficient is defined as:

$$
\begin{aligned}
k_{e f f} & =R_{T_{\|}}^{k} /\left(-n_{e} \nabla_{\|} T_{e}\right) \\
& =\int m_{e} v^{\prime}\left(\frac{\partial f^{\mathrm{k}}}{\partial t}\right)_{\text {coll. }} d \vec{v} /\left(-n_{e} \nabla_{\|} T_{e}\right)
\end{aligned}
$$

In a coupling steady state (which means that SOLPS runs, with the modified kinetic factors that are calculated in KIPP based on the profiles from the previous SOLPS run, to reach convergence and the profiles out of it are the same as those from the previous run) where the time-dependent terms can be dropped, the electron perpendicular particle flux in SOLPS is automatically included in the particle source in KIPP:

$$
S_{p}^{\mathrm{k}}=S_{p}-\nabla_{\perp} \Gamma_{e \perp}
$$

\section{Appendix B.3.2. Effective heat conduction coefficient $c_{\text {eff }}$}

The kinetic factor, heat conduction coefficient $c_{e}$, exists in the electron energy conservation equation, however, a difficult point needs to be clarified when calculating the effective one, since the internal electron energy equation (Eq. (B.3)) is solved in SOLPS. In order to be compared with Eq. (B.3), subtracting Eq. (B.8) multiplied by $u_{e \|}^{\mathrm{k}}$ from Eq. (B.9) leads to:

$$
\frac{\partial}{\partial t}\left(\frac{3}{2} n_{e}^{\mathrm{k}} T_{e}^{\mathrm{k}}\right)+\nabla_{\|}\left(q_{e \|}^{\mathrm{k}}-n_{e}^{\mathrm{k}} T_{e}^{\mathrm{k}} u_{e \|}^{\mathrm{k}}\right)=-n_{e}^{\mathrm{k}} T_{e}^{\mathrm{k}} \nabla_{\|} u_{e \|}^{\mathrm{k}}+Q_{c}^{\mathrm{k}}-u_{e \|}^{\mathrm{k}} R_{T_{\|}}^{\mathrm{k}}+S_{E e}^{\mathrm{k}}
$$

By comparing Eq. (B.3) with Eq. (B.18), the profile of the effective heat conduction coefficient can be obtained in KIPP as:

$$
\begin{aligned}
c_{e f f} & =\left(q_{e \|}^{\mathrm{k}}-n_{e}^{\mathrm{k}} T_{e}^{\mathrm{k}} u_{e \|}^{\mathrm{k}}-\frac{3}{2} n_{e} T_{e} u_{e \|}\right) /\left(-n_{e} \tau_{e} \frac{T_{e}}{m_{e}} \nabla_{\|} T_{e}\right) \\
& =\left(\frac{1}{2} m_{e} \int f_{e}^{\mathrm{k}} v^{2} v_{\|} d \vec{v}-\frac{5}{2} n_{e} T_{e} u_{e \|}\right) /\left(-n_{e} \tau_{e} \frac{T_{e}}{m_{e}} \nabla_{\|} T_{e}\right)
\end{aligned}
$$

In the coupling steady state, the perpendicular heat flux is automatically included in the energy source term:

$$
S_{E_{e}}^{\mathrm{k}}=S_{E_{e}}-\nabla_{\perp} q_{e \perp}
$$


Appendix B.3.3. Effective sheath potential drop $\Delta \phi_{\text {eff }}$

Now we deal with the implementation of kinetic effects into the boundary conditions. The two boundary coefficients: the sheath potential drop and electron sheath heat transmission coefficient in SOLPS, are obtained by assuming a cut-off Maxwellian distribution function at the boundary (sheath edge). As pointed out in section 3.2, KIPP can give a self-consistent distribution function (Eq. (9)) at the boundary without solving Poisson's equation for the Debye sheath. A critical velocity $v_{c}$ can thus be achieved, which corresponds to a floating potential drop through the sheath which gives ambipolar flux. Hence an effective potential drop can be obtained:

$$
\Delta \phi_{e f f}=\frac{m_{e} v_{c}^{2}}{2 e}
$$

The parallel heat flux density through the boundary (or at the target), calculated in KIPP, is:

$$
q_{t \|}^{\mathrm{k}}=\frac{1}{2} m_{e} \int_{v_{c}}^{\infty} f_{t}^{\mathrm{k}} v^{2} v_{\|} d \vec{v}
$$

In later discussions, "boundary" and "target" have the same meaning: the right end of the simulation boundary. The left end is the stagnation point (more details about the geometry can be found in section 5.1).

Appendix B.3.4. Effective electron sheath heat transmission coefficient $\gamma_{\text {eff }}$

The default boundary condition for the internal electron energy conservation equation (Eq. (B.3)) in SOLPS is applied as an energy sink at the guard cell $n x$ shown in Fig. B.28. It is not straightforward to directly calculate the effective electron sheath heat transmission coefficient $\gamma_{\text {eff }}$ in KIPP. In the following discussions of this subsection we first analyse the energy balance at cells adjacent to the target and then implement the adaptation of the SOLPS boundary condition. Based on the adapted boundary condition, we then derive the formula for calculating the effective electron sheath heat transmission coefficient in KIPP.

Energy balance of the guard cell in SOLPS. We first analyse the energy balance of cell $n x$ (the guard cell) in a SOLPS run. The heat fluxes on the left and right 


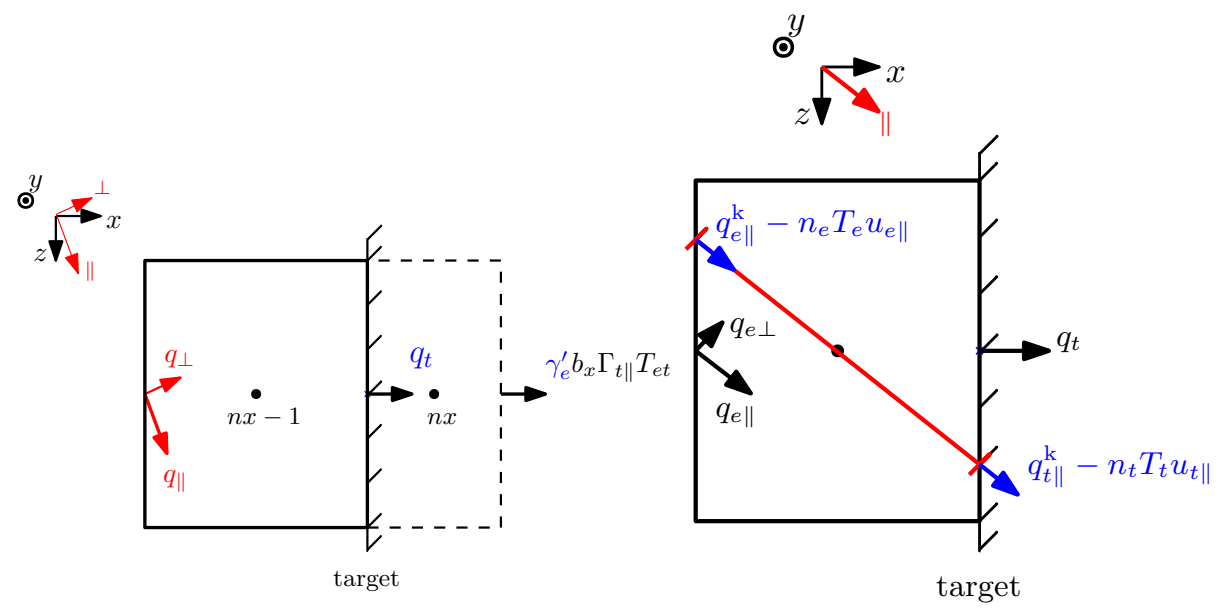

Figure B.28: In the default SOLPS run, the bound- Figure B.29: Electron heat flux densities ary electron heat flux is determined by the user- through faces of the cell $n x-1$ in SOLPS specified constant $c_{1}$ or $\gamma_{e}^{\prime}\left(\gamma_{e}^{\prime}=c_{1}+|e \Delta \phi| / T_{e t}\right)$. (in black) and of the corresponding cell So $q_{t}=\gamma_{e}^{\prime} b_{x} \Gamma_{t \|} T_{e t}$. smax in KIPP (in blue). In a coupling run, $q_{t}$ is supposed to be given based on the self-consistent boundary heat flux density $q_{t \|}^{\mathrm{k}}$.

faces of the guard cell are balanced, implying that the poloidal heat flux density through the boundary is specified, as shown in Fig. B.28, as:

$$
\begin{aligned}
q_{t} & =\gamma_{e}^{\prime} b_{x} \Gamma_{t \|} T_{e t} \\
& =\left(c_{1}+\frac{|e \Delta \phi|}{T_{e t}}\right) b_{x} \Gamma_{t \|} T_{e t}
\end{aligned}
$$

where $\Gamma_{t \|}$ and $T_{e t}$ are electron particle flux density and electron temperature at the boundary, $c_{1}$ is a user-specified constant. $\gamma_{e}^{\prime}$ is a coefficient, defined as:

$$
\gamma_{e}^{\prime} \equiv c_{1}+\frac{|e \Delta \phi|}{T_{e t}}
$$

It is not the electron sheath heat transmission coefficient. The relation between the coefficient $\gamma_{e}^{\prime}$ specified for the SOLPS default boundary condition and the electron sheath heat transmission coefficient will be discussed below.

Energy balance of the cell adjacent to the target in SOLPS. We then analyse the energy balance of cell $n x-1$ in the steady state of a SOLPS run. One 
should note again that the electron heat flux density in the poloidal direction is defined according to Eq. (A.4) at cell faces for internal cells, but defined as $q_{t}$ at the boundary. Based on Eq. (B.3), in the steady state, at cell $n x-1$, as shown in Fig. B.28, the energy for electrons is balanced by heat flux densities through the left and right faces of this cell, and the terms on the right hand side of Eq. (B.28):

$$
\begin{aligned}
& \frac{1}{h_{x}}\left[q_{t}-\left(\left(\frac{3}{2} n_{e} T_{e} u_{e \|}+q_{e \|}^{\text {cond }}\right) b_{x}+q_{e \perp} b_{z}\right)_{l}\right] \\
& =-n_{e} T_{e} \nabla_{\|} u_{e \|}-Q_{\Delta}+S_{E e}
\end{aligned}
$$

where $h_{x}$ is the poloidal length of cell $n x-1$ and the subscript $l$ denotes the variable calculated at the left face of cell $n x-1$. As it can be clearly seen, the electron heat flux density at the left face still has the form of Eq. (A.4), but the boundary heat flux density $q_{t}$, determined by $\gamma_{e}^{\prime}$ (Eq. B.23), cannot be given self-consistently within the fluid model, as discussed in section 3.1. So, $q_{t}$ remains to be replaced with the one calculated in KIPP.

Energy balance of the cell adjacent to the target in KIPP. Similarly, in the steady state of a KIPP run, the energy balance can be achieved based on Eq. (B.18) at the corresponding cell (cell smax), as shown in Fig. B.29:

$$
\begin{aligned}
& \frac{1}{h_{\|}}\left[\left(q_{t \|}^{\mathrm{k}}-\Gamma_{t \|} T_{e t}\right)-\left(q_{e \|}^{\mathrm{k}}-\Gamma_{e \|} T_{e}\right)_{l}\right] \\
& =-n_{e}^{\mathrm{k}} T_{e}^{\mathrm{k}} \nabla_{\|} u_{e \|}^{\mathrm{k}}+Q_{c}^{\mathrm{k}}-u_{e \|}^{\mathrm{k}} R_{T_{\|}}^{\mathrm{k}}+S_{E e}^{\mathrm{k}}
\end{aligned}
$$

The parallel length of the cell in KIPP $h_{\|}=h_{x} / b_{x}$. The subscript $l$ denotes the variable calculated at the left face of this cell. The details of the geometry and the relation between the grids generated in SOLPS and KIPP can be found in sections 5.1.1 and 5.1.2. For simplicity, by comparing Eqs. (B.25) and (B.26), one can specify:

$$
q_{t}=b_{x}\left(q_{t \|}^{\mathrm{k}}-\Gamma_{t \|} T_{e t}\right)
$$

to incorporate the kinetic boundary condition into SOLPS. In the coupling steady state, the additional heat flux density $q_{e \perp} b_{z}$ in SOLPS at the left face 
will be included in the automatic source term $S_{E e}^{\mathrm{k}}$ in KIPP. However, Eq. (B.27) is not used in this work since the term $q_{e} b_{z}$ is present in Eq. (B.25), but not explicitly in Eq. (B.26). Eq. (B.27) would result in an inconsistent source term for cell smax in KIPP. An adaptation of the SOLPS boundary condition can avoid this.

Before describing the adaptation of SOLPS boundary condition, one point, which we did not mention above because it would only add difficulty to discussions, has to be clarified for following discussions. The boundary electron heat flux density mentioned above is defined, in the SOLPS code, in the same form as for internal cells. It consists of two parts:

$$
q_{t}=b_{x} q_{t \|}+b_{z} q_{t \perp}
$$

where $q_{t \|}$ and $q_{t \perp}$ are defined in the same forms as those at the internal cell faces but calculated based on parameters in cell $n x-1$ and the guard cell $n x$. This will be discussed more in detail below.

Adaptation of the SOLPS boundary condition to the coupling scheme. As mentioned above, the boundary electron heat flux density in SOLPS still has the form of Eq. (A.4): $q_{t}=b_{x} q_{t \|}+$ $b_{z} q_{t \perp}$, as shown in Fig. B.30. In order to be compatible with the internal cells and have the same form of the source (Eq. (B.20)), the SOLPS boundary is modified as (see Fig. B.30):

$$
\begin{array}{r}
b_{x} q_{t \|}+b_{z} q_{t \perp}=\gamma_{e \|}^{\prime} b_{x} \Gamma_{t \|} T_{e t}+b_{z} q_{t \perp} \\
\longrightarrow \quad q_{t \|}=\gamma_{e \|}^{\prime} \Gamma_{t \|} T_{e t}
\end{array}
$$

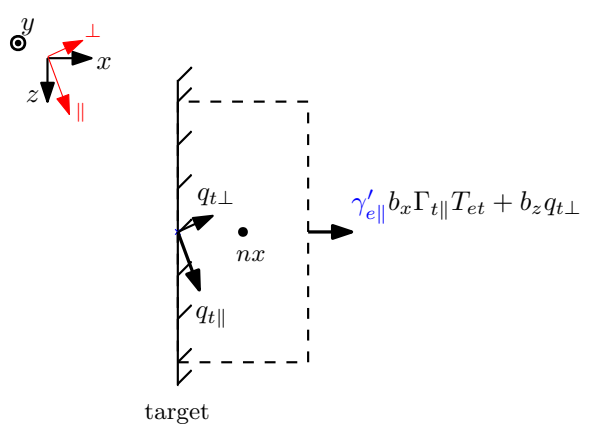

Figure B.30: In the default boundary condition illustrated in Fig. B.28, the boundary electron heat flux density is specified as $q_{t}=b_{x} q_{t \|}+b_{z} q_{t \perp}=\gamma_{e}^{\prime} b_{x} \Gamma_{t \|} T_{e t}$. However, in the adapted boundary condition here, it is specified as $q_{t}=b_{x} q_{t \|}+b_{z} q_{t \perp}=$ $\gamma_{e \|}^{\prime} b_{x} \Gamma_{t \|} T_{e t}+b_{z} q_{t \perp}$.

Instead of specifying the constant $c_{1}$ in Eq. (B.23), the new boundary condi- 
tion only specifies the parallel part, and then the perpendicular part is determined automatically since $q_{t \perp} / q_{t \|} \propto b_{z} \chi_{e \perp} / b_{x} \chi_{e \|}$. Subtracting $\nabla_{\perp} q_{e \perp}$ from both sides of Eq. (B.25) and substituting Eq. ( B.29) into it, leads to:

$$
\begin{aligned}
& \frac{1}{h_{\|}}\left[\gamma_{e \|}^{\prime} \Gamma_{t \|} T_{e t}-\left(\frac{3}{2} n_{e} T_{e} u_{e \|}+q_{e \|}^{\text {cond }}\right)_{l}\right] \\
& =-n_{e} T_{e} \nabla_{\|} u_{e \|}-Q_{\Delta}+S_{E e}-\nabla_{\perp} q_{e \perp}
\end{aligned}
$$

The adapted SOLPS boundary condition (specifying $\gamma_{e \|}^{\prime}$ ) is used in the following coupling runs.

By comparing Eqs. (B.26) and (B.31), one can derive:

$$
\begin{aligned}
\gamma_{\|, e f f}^{\prime} & =\frac{q_{t \|}^{\mathrm{k}}-\Gamma_{t \|} T_{e t}}{\Gamma_{t \|} T_{e t}} \\
& =\frac{q_{t \|}^{\mathrm{k}}}{\Gamma_{t \|} T_{e t}}-1 \\
& =\gamma_{e f f}-1
\end{aligned}
$$

where the effective electron sheath heat transmission coefficient is defined as:

$$
\begin{aligned}
\gamma_{e f f} & =\frac{q_{t \|}^{\mathrm{k}}}{\Gamma_{t \|} T_{e t}} \\
& =\frac{1}{2} m_{e} \int_{v_{c}}^{\infty} f_{t}^{\mathrm{k}} v^{2} v_{\|} d \vec{v} /\left(\Gamma_{t \|} T_{e t}\right)
\end{aligned}
$$

In the coupling steady state, Eq. (B.20) is automatically fulfilled.

The difference of unity between $\gamma_{\|, \text {eff }}^{\prime}$ and $\gamma_{\text {eff }}$ in Eq. (B.32) is attributed to the fact that the "internal" electron energy conservation equation, as a result of subtracting $\nabla_{\|}\left(n_{e} T_{e} u_{e \|}\right)$ from both sides of the total electron energy conservation equation, is used in SOLPS. For simplicity, the following discussions in this work are based on the total electron energy conservation equation where the electron sheath heat transmission coefficient is $\gamma_{e \|}$, and $\frac{5}{2}$ is the coefficient for the convective heat flux (instead of $\gamma_{e \|}^{\prime}$ and $\frac{3}{2}$ ):

$$
\gamma_{e \|}=\gamma_{e \|}^{\prime}+1
$$

In a coupling run, $\gamma_{e \|}$ is replaced by $\gamma_{e f f}$. Since the perpendicular terms have been regarded as source terms, in later discussions, heat flux density through the cell faces or the boundary only means the parallel part $\left(q_{e \|}\right.$ and $\left.q_{t \|}\right)$. 
Appendix B.4. Replacing kinetic factors with the effective ones in SOLPS

The effective heat conduction coefficient $c_{e f f}$, effective thermal force coefficient $k_{e f f}$, effective potential drop $\Delta \phi_{\text {eff }}$ and effective sheath heat transmission coefficient $\gamma_{\text {eff }}$ mentioned above are obtained when KIPP reaches a quasi-steady state, since a steady state like the one in a fluid code is not possible in a kinetic code (this will be discussed in section 5.2), which here means that the heat flux through each cell face is not changing much with time, that is, the profile of $c_{e f f}$ and boundary $\gamma_{e f f}$ are not changing much with time. Then the effective coefficients are transferred back into SOLPS, replacing $c_{e}, k_{\|}, \Delta \phi$ and $\gamma_{e \|}$ in Eqs. (B.4), (B.5), (5) and (B.34).

\section{References}

[1] R. Schneider, et al., Plasma edge physics with b2-eirene, Contrib. Plasma Phys. 46 (2006) 3.

[2] S. I. Braginskii, Reviews of Plasma Physics, Vol. 1, Consultants Bureau, New York, 1965, Ch. Transport Processes In A Plasma, p. 205.

[3] F. F. Chen, Introduction To Plasma Physics and Controlled Fusion, Plenum Press, New York and London, 1984.

[4] R. J. Goldston, Energy confinement scaling in tokamaks: some implications of recent experiments with ohmic and strong auxilliary heating, Plasma Physics and Controllel Fusion 26 (1984) 87.

[5] J. Wesson, Tokamaks, Clarendon Press, Oxford, United Kingdom, 2004.

[6] R. Chodura, Basic problems in edge plasma modelling, Contrib. Plasma Phys. 28 (1988) 303.

[7] R. Chodura, Nonlocal heat flux in the scrape-off layer of a high-temperature plasma, Contrib. Plasma Phys. 28 (1988) 325.

[8] R. Chodura, Non-local heat conduction along a scrape-off layer with strong recycling, Contrib. Plasma Phys. 30 (1990) 153. 
[9] R. Chodura, Kinetic effects in the scrape off layer, Contrib. Plasma Phys. $32(1992) 219$.

[10] P. C. Stangeby, The Plasma Boundary of Magnetic Fusion Devices, Institute of Physics, Bristol, UK, 2000.

[11] S. I. Krasheninnikov, Superthermal particles and electron thermal conductivity, Sov. Phys. JETP 67 (1988) 2483.

[12] S. I. Krasheninnikov, On nonlocal electron heat conduction, Physics of Plasmas 5 (1993) 74.

[13] J. R. Albritton, E. A. Williams, I. B. Bernstein, K. P. Swartz, Nonlocal electron heat transport by not quite maxwell-boltzmann distributions, Phys. Rev. Lett. 57 (1986) 1887.

[14] A. M. Mirza, et al., Heat transport formula in strongly inhomogeneous plasmas, Physics Letters A 141 (1989) 56.

[15] G. Murtaza, et al., Delocalized heat flux for low z plasmas, Physics Letters A 144 (1990) 164.

[16] P. J. Catto, K. Yeoh, Long mean free path modifications of electron heat conduction, Contrib. Plasma Phys. 38 (1998) 207.

[17] P. J. Catto, M. Grinneback, Electron heat conduction modifications due to long mean free path effects, Physics Letters A 277 (2000) 323.

[18] E. Zawaideh, F. Najmabadi, R. W. Conn, Generalized fluid equations for parallel transport in collisional to weakly collisional plasmas, Physics of Fluids 29 (1986) 463.

[19] E. Zawaideh, F. Najmabadi, R. W. Conn, Effects of pressure anisotropy on plasma transport, Physics of Fluids 29 (1986) 3702.

[20] E. Zawaideh, N. S. Kim, Generalized parallel heat transport equations in collisional to weakly collisional plasmas, Physics of Fluids 31 (1988) 3280. 
[21] G. J. Radford, The application of moment equations to scrape off layer plasmas, Contrib. Plasma Phys. 32 (1992) 297.

[22] G. J. Radford, A. V. Chankin, et al., The particle and heat drift fluxes and their implementation into the edge2d transport code, Contrib. Plasma Phys. 36 (1996) 187.

[23] G. J. Radford, et al., The development of simplified heat flux limiters for edge2d, Contrib. Plasma Phys. 38 (1998) 183.

[24] Y. L. Igitkhanov, P. N. Yushmanov, Non-local transport in the scrape-off tokamak plasma, Contrib. Plasma Phys. 28 (1988) 341.

[25] Y. L. Igitkhanov, A. Y. Pigarov, Non-local transport effects on the tokamak sol plasma parameters, Journal of Nuclear Materials 176 (1990) 557.

[26] A. Bergmann, et al., Implementation into b2 of a 21-moment description for the parallel transport, Contrib. Plasma Phys. 36 (1996) 192.

[27] W. Fundamenski, Parallel heat flux limits in the tokamak scrape-off layer, Plasma Phys. Control. Fusion 47.

[28] J. F. Luciani, P. Mora, J. Virmont, Nonlocal heat transport due to steep temperature gradients, Phys. Rev. Lett. 51 (1983) 18.

[29] J. R. Albritton, et al., Nonlocal electron heat transport by not quite maxwellian-boltzmann distribution, Physical Review Letters 57 (1986) 1887.

[30] B. P. Schurtz, P. D. Nicolai, M. Busquet, A nonlocal electron conduction model for multidimensional radiation hydrodynamics codes, Physics of Plamsas 7 (2000) 4238.

[31] J. T. Omotani, B. D. Dudson, A nonlocal electron conduction model for multidimensional radiation hydrodynamics codes, Plasma Phys. Control. Fusion 55 (2013) 055009. 
[32] M. Zhao, A. V. Chankin, D. P. Coster, Kinetic simulations of electron heat flux in the scrape-off layer, Nuclear Materials and Energy 12 (2017) 819.

[33] O. V. Batishchev, S. I. Krasheninnikov, et al., Kinetic effects in tokamak scrape-off layer plasmas, Phys. Plasmas 4 (1997) 1672.

[34] A. Froese, T. Takizuka, M. Yagi, Pic simulation study of heat transport kinetic factors in scrape-off layer plasmas, Contrib. Plasma Phys. 52 (2012) 534.

[35] K. Kupfer, et al., Kinetic modeling of scrapeoff layer plasmas, Phys. Plasmas 3 (1966) 3644.

[36] D. Tskhakaya, et al., On kinetic effects during parallel transport in the sol, Contrib. Plasma Phys. 48 (2008) 89.

[37] D. Tskhakaya, et al., Kinetic simulations of the parallel transport in the jet scrape-off layer, Journal of Nuclear Material 390 (2009) 335.

[38] C. K. Birdsall, A. B. Langdon, Plasma Physics via Computer Simulation, McGraw-Hill, New York, 1985.

[39] R. J. LeVeque, Finite-Volume Method for Hyperbolic Problems, Cambridge University Press, Cambridge, United Kingdom, 2004.

[40] T. Takizuka, H. Abe, A binary collision model for plasma simulation with a particle code, J. Comput. Phys. 25 (1977) 205.

[41] T. Takizuka, K. Tani, M. Azumi, K. Shimizu, Particle simulation of divertor plasma, J. Nucl. Mater. 128 (1984) 104.

[42] T. Takizuka, Development of the parasol code and full particle simulation of tokamak plasma with an open-field sol-divertor region using parasol, Plasma Science and Technology 12 (2011) 316.

[43] T. Takizuka, Kinetic effects in edge plasma: kinetic modeling for edge plasma and detached divertor, Plasma Physics and Controlled Fusion 59 (2017) 034008. 
[44] A. Froese, Kinetic analysis of parallel heat transport in scrape-off layer plasmas using a particle simulation, Ph.D. thesis, Kyushu University, Kyushu (2010).

[45] A. Froese, T. Takizuka, M. Yagi, Effect of source and sink on heat transport in the sol, Contrib. Plasma Phys. 50 (2010) 273.

[46] A. Froese, T. Takizuka, M. Yagi, Kinetic particle simulation study of parallel heat transport in scrape-off layer plasmas over a wide range of collisionalities, Plasma and Fusion Research 5 (2010) 26.

[47] A. Froese, T. Takizuka, M. Yagi, Electron parallel heat transport in the scrape-off layer using a particle-in-cell code, Plasma and Fusion Research 5.

[48] M. Hosokawa, et al., Kinetic modelling of divertor fluxes during elms in iter and effect of in/out divertor plasma asymmetries, Plasma and Fusion Research 11 (2016) 1403104.

[49] D. Tskhakaya, R. Schneider, Optimization of pic codes by improved memory management, Journal of Computational Physics 225 (2007) 829.

[50] D. Tskhakaya, et al., The particle-in-cell method, Contib. Plasma Phys. 47 (2007) 563.

[51] D. Tskhakaya, et al., On kinetic effects during parallel transport in the sol, Contib. Plasma Phys. 48 (2008) 89.

[52] D. Tskhakaya, On recent massively parallelized pic simulations of the sol, Contib. Plasma Phys. 52 (2012) 490.

[53] D. Tskhakaya, et al., 1d kinetic modelling of the jet sol with tungsten divertor plates, Journal of Nuclear Materials 438.

[54] D. Tskhakaya, et al., Modelling of tungsten re-deposition coefficient, Journal of Nuclear Materials 463 (2015) 624. 
[55] D. Tskhakaya, et al., Stability of the tonkslangmuir discharge pre-sheath, Physics of Plasmas 23 (2016) 032128.

[56] D. Tskhakaya, Kinetic modelling of the plasma recombination, Contrib. Plasma Phys. 56 (2016) 698.

[57] D. Tskhakaya, One-dimensional plasma sheath model in front of the divertor plate, Plasma Phys. Control. Fusion 59 (2017) 114001.

[58] O. V. Batishchev, et al., Influence of kinetic effects on particle and energy flows in the iter divertor, Contrib. plasma Phys. 34 (1994) 436.

[59] O. V. Batishchev, et al., Kinetic effects on particle and heat fluxes in detached plasmas, Phys. Plasmas 3 (1996) 3386.

[60] O. V. Batishchev, et al., Kinetic modelling of detached and elmy sol plasmas, Contributions to Plasma Physics 36 (1996) 225.

[61] M. A. Dorf, R. H. Cohen, et al., Progress with the cogent edge kinetic code: Collision operator options, Contrib. Plasma Phys. 52 (2012) 518.

[62] M. A. Dorf, R. H. Cohen, et al., Progress with the cogent edge kinetic code: Implementing the fokker-planck collision operator, Contrib. Plasma Phys. $54(2014) 517$.

[63] M. A. Dorf, M. R. Dorr, et al., Continuum kinetic modeling of the tokamak plasma edge, Phys. Plasmas 23 (2016) 056102.

[64] P. L. Bhatnagar, E. P. Gross, M. Krook, A model for collision processes in gases. i. small amplitude processes in charged and neutral one-component systems, Physical Review 94 (1954) 511.

[65] B. A. Trubnikov, Reviews of Plasma Physics, Vol. 1, Consultants Bureau, New York, 1965, Ch. Particle Interactions in A Fully Ionized Plasma, p. 105. 
[66] A. S. Kukushkin, A. M. Runov, Implementation of non-local transport model into 2d fluid code, Contrib. Plasma Phys. 34 (1994) 204.

[67] Y. L. Igitkhanov, A. S. Kukushkin, A. M. Runov, Application of bgk collision operator for kinetic correction of fluid models, Contrib. Plasma Phys. 34 (1994) 216.

[68] Y. L. Igitkhanov, A. M. Runov, Implication of kinetic effects for fluid codes, Contrib. Plasma Phys. 34 (1994) 221.

[69] Y. L. Igitkhanov, A. M. Runov, Non-local sheath boundary conditions for fluid equations, Contrib. Plasma Phys. 34 (1994) 204.

[70] A. V. Chankin, D. P. Coster, G. Meisl, Development and benchmarking of a new kinetic code for plasma periphery (kipp), Contrib. Plasma Phys. 52 (2012) 500.

[71] A. V. Chankin, D. P. Coster, Benchmarks of kipp: Vlasov-fokker-planck code for parallel plasma transport in the sol and divertor, Contrib. Plasma Phys. 54 (2014) 493.

[72] A. V. Chankin, D. P. Coster, On the locality of parallel transport of heat carrying electrons in the sol, Journal of Nuclear Materials 463 (2015) 498.

[73] G. Meisl, A. V. Chankin, D. P. Coster, Kinetic modelling of temperature equilibration rates in the plasma, Journal of Nuclear Materials 438.

[74] D. Coster, et al., Extensions to the solps edge plasma simulation code to include additional surface interaction posibilities, Physica Scripta 2006 (2006) 9.

[75] M. Zhao, Towards a more complete description of tokamak edge plasmas: Solps simulations with kinetic electron effects, Ph.D. thesis, Technische Universität München, München, https://pure.mpg.de/rest/items/item_2590992/component/file_2590995/content (2018). 
[76] D. P. Coster, Whole device elm simulations, Journal of Nuclear Materials $390(2009) 826$.

[77] D. P. Coster, Reduced physics models in solps for reactor scoping studies, Contrib. Plasma Phys. 56 (2016) 790.

[78] D. Bohm, The Characteristics of Electrical Discharges in Magnetic Fields, McGraw-Hill, New York, 1949.

[79] Y. L. Igitkhanov, A. M. Runov, Non-local sheath boundary conditions for fluid equations, Contributions to Plasma Physics 32 (1992) 308.

[80] J. A. Wesson, Effect of temperature gradient on plasma sheath, Plasma Phys. Control. Fusion 37 (1995) 1459.

[81] P. C. Stangeby, A problem in the interpretation of tokamak langmuir probes when a fast electron component is present, Plasma Phys. Control. Fusion 37 (1995) 1031.

[82] J. A. Tagle, P. C. Stangeby, S. K. Erents, Errors in measuring electron temperatures using a single langmuir probe in a magnetic field, Plasma Phys. Control. Fusion 29 (1987) 297.

[83] J. Horacek, et al., Predicted effects of parallel temperature gradients on the overestimation of tcv divertor target langmuir probe t-e measurements, Journal of Nuclear Materials 313 (2003) 931.

[84] D. Tskhakaya, et al., Interpretation of divertor langmuir probe measurements during the elms at jet, Journal of Nuclear Materials 415.

[85] P. C. Stangeby, Langmuir-, and bolometer-probe interpretation for plasmas with two electron components, Journal of Nuclear Materials 128 (1984) 969.

[86] M. A. Jaworski, et al., Modification of the electron energy distribution function during lithium experiments on the national spherical torus experiment, Fusion Engineering and Design 87 (2012) 1711. 
[87] J. G. Watkins, et al., An evaluation of kinetic effects in the diii-d divertor, Journal of Nuclear Materials 266.

[88] S. Marsen, et al., Experimental sheath heat transmission factors in diverted plasmas in jet, Journal of Nuclear Materials 438.

[89] I. Duran, et al., Assessment of the effect of parallel temperature gradients in the jet sol on te measured by divertor target langmuir probes, Journal of Nuclear Materials 463 (2015) 432.

[90] M. M. Shoucri, R. Gagn, Splitting schemes for the numerical solution of a two-dimensional vlasov equation, Journal of Computational Physics 27 (1978) 315.

[91] O. V. Batishchev, et al., Journal of Plasma Physics 61 (1999) 347.

[92] S. E. Parker, R. J. Procassini, C. K. Birdsall, B. I. Cohen, A suitable boundary condition for bounded plasma simulation without sheath resolution, Journal of Computational Physics 104 (1993) 41.

[93] A. Abdulle, et al., The heterogeneous multiscale method, Acta Numerica $21(2012) 1$.

[94] SOLPS user manual, https://solps-mdsplus.aug.ipp.mpg.de/solps.pdf.

[95] M. Zhao, A. V. Chankin, D. P. Coster, SOLPS simulations with electron kinetic effects, accepted by Plasma Physics and Controlled Fusion. 